\title{
Exploring the potential of microwave diagnostics in SEP forecasting: The occurrence of SEP events
}

\author{
Pietro Zucca ${ }^{1}$, Marlon Núñez ${ }^{2}$, and Karl-Ludwig Klein ${ }^{1, *}$ \\ 1 LESIA-UMR 8109 - Observatoire de Paris, PSL Research University, CNRS, Université P \& M Curie and Paris-Diderot, \\ 92190 Meudon, France \\ ${ }^{*}$ Corresponding author: ludwig.klein@obspm.fr \\ 2 Departamento de Lenguajes y Ciencias de la Computación, Universidad de Málaga, Campus de Teatinos, 29071 Málaga, Spain
}

Received 17 January 2017 / Accepted 13 April 2017

\begin{abstract}
Solar energetic particles (SEPs), especially protons and heavy ions, may be a space-weather hazard when they impact spacecraft and the terrestrial atmosphere. Forecasting schemes have been developed, which use earlier signatures of particle acceleration to predict the arrival of solar protons and ions in the space environment of the Earth. The UMASEP (University of MAlaga Solar particle Event Predictor) scheme forecasts the occurrence and the importance of an SEP event based on combined observations of soft X-rays, their time derivative and protons above $10 \mathrm{MeV}$ at geosynchronous orbit. We explore the possibility to replace the derivative of the soft X-ray time history with the microwave time history in the UMASEP scheme. To this end we construct a continuous time series of observations for a 13-month period from December 2011 to December 2012 at two microwave frequencies, 4.995 and $8.8 \mathrm{GHz}$, using data from the four Radio Solar Telescope Network (RSTN) patrol stations of the US Air Force, and feed this time series to the UMASEP prediction scheme. During the selected period the Geostationary Operational Environmental Satellites (GOES) detected nine SEP events related to activity in the western solar hemisphere. We show that the SEP forecasting using microwaves has the same probability of detection as the method using soft X-rays, but no false alarm in the considered period, and a slightly increased warning time. A detailed analysis of the missed events is presented. We conclude that microwave patrol observations improve SEP forecasting schemes that employ soft X-rays. High-quality microwave data available in real time appear as a significant addition to our ability to predict SEP occurrence.
\end{abstract}

Key words. Sun: particle emission - Sun: radio radiation - Solar-terrestrial relations

\section{Introduction}

Solar energetic particles (SEPs), especially protons and heavy ions, can disturb or damage electronic equipment aboard spacecraft, affect the ionization and chemistry of the high terrestrial atmosphere, and create secondaries that interact with equipment and living beings aboard aircraft. SEPs may be a major space-weather hazard and a fundamental concern to manned spaceflight. Forecasting the occurrence and importance of an SEP event is therefore a task for space-weather research, and appears mandatory if human beings are to be sent aboard spacecraft beyond low-Earth orbit. SEPs are accelerated in relationship with major eruptive events in the corona, flares and coronal mass ejections (CMEs).

As of today, it is not possible to reliably predict a flare or a CME. It is also not possible to predict before the eruptive event whether it will lead to a major SEP event or not. The only practicable forecasting strategy is presently to infer the SEPs to come from the first observations of the eruptive activity in the corona or from early signatures of fast particles themselves. Several different, but complementary approaches have been developed. Some use the analysis of solar electromagnetic radiation as the basic ingredient. Because of their continuous availability, soft X-ray observations by the Geostationary Operational Environmental Satellites (GOES) of NOAA play a key role in these forecasting schemes.
The empirical forecast systems of the US Air Force (USAF; Smart \& Shea 1992; Kahler et al. 2007) and of the NOAA Space Weather Prediction Center (Balch 2008) are based on the location of the flare and the importance and time evolution of the associated soft X-ray burst. The USAF system predicts the onset, rise time and peak of the SEP event at several energies above $5 \mathrm{MeV}$, radiation dose rates in the terrestrial atmosphere and ionospheric absorption. The NOAA system uses in addition the occurrence of metre-wave radio emission related to CMEs and shocks. It predicts the probability of occurrence of an SEP event, the maximum intensity and its time. Both schemes are semi-automatic, in that operators are supposed to use them for a final decision on whether an event is to be predicted or not. Laurenza et al. (2009) added an observational criterion of the escape of particles accelerated in the corona to the interplanetary space, using the observation of decametric-to-kilometric radio emission from electron beams that travel through the high corona (type III bursts). Garcia (2004), Belov (2009) and the COronal Mass Ejections and Solar Energetic Particles (COMESEP) model (Dierckxsens et al. 2015) propose methods that calculate the probability of SEP events from X-ray observations. The empirical and operational SEP forecasting methods using electromagnetic observations of solar activity currently rely more on data related to the flare rather than the CME-driven shock to predict well-connected SEP events. 
Physics-based SEP forecasting models have so far been mostly developed based on shock acceleration theories or on particle transport modelling, assuming injections into the interplanetary space from an unspecified generic accelerator. These models are not operational yet. Physics-based particle models like SOLPENCO ${ }^{1}$ (Aran et al. 2006, 2008) and SPARX Marsh et al. (2015) are able to make a post-event prediction of the SEP intensity profiles. The core of SOLPENCO contains a database of pre-calculated synthetic flux profiles of gradual proton events for different interplanetary scenarios for energies up to $200 \mathrm{MeV}$. SPARX uses a pre-generated database of model runs containing varying proton injection locations for energies in the ranges $E>10 \mathrm{MeV}$ and $E>60 \mathrm{MeV}$.

When SEP forecasting is based exclusively on solar radiative signatures, there is no certainty whether the Earth or the spacecraft of interest is magnetically connected to the particle accelerator or not. The location of the eruptive activity is only a partial indicator. The problem is avoided by forecasting schemes based on in situ observations of energetic particles themselves. The longest warning times are achieved when the particles employed are particularly fast. The RELEASE system (Posner 2007) uses energetic electrons, while the ground-level enhancement (GLE)-Alert system (Souvatzoglou et al. 2014) is based on relativistic protons observed by neutron monitors.

The UMASEP (University of Malaga Solar Energetic Particle) scheme (Núñez 2011) combines the monitoring of solar soft X-ray emission, its time derivative and solar protons, using GOES measurements. Simultaneous rises in the soft $\mathrm{X}$-ray flux and the particle intensity are considered as an indicator that an SEP event is to occur. We conduct an exploratory study to see if the soft X-ray data can be replaced or complemented by microwave observations referring to the gyrosynchrotron emission of mildly relativistic electrons accelerated in the associated flare. The motivation is twofold: from a physics viewpoint, microwave emission produced by non-thermal electrons may be expected to be more closely related to SEP acceleration than soft X-rays, which are emitted by the plasma heated during the solar eruption. From an empirical viewpoint, the derivative of the soft X-ray time profile is known to mimic the time profile of microwave emission from non-thermal electrons. The UMASEP scheme and the microwave emission are briefly introduced in Section 2. In Section 3 composite profiles of microwave flux densities during a 13-month interval are presented, and the results of a run of UMASEP with these data are described. The reasons for erroneous predictions are studied in detail in Section 4. The usefulness of microwave data is discussed in the light of these results in Section 5.

\section{The UMASEP prediction scheme and microwave burst emission}

\subsection{The UMASEP model for well-connected SEP events}

The UMASEP scheme (Núñez 2011) comprises two different procedures to forecast SEP events, which are referred to as "well-connected" and "poorly-connected" prediction models. The prediction model of "well-connected" events uses the common rise, with a plausible time delay, of the soft X-ray flux of the Sun and the intensities of protons in each of the energy channels measured by the GOES particle detectors, i.e. 9-500 MeV. The correlated occurrence of the two rises is

\footnotetext{
${ }^{1}$ http://dev.sepem.oma.be/help/solpenco2_intro.html
}

considered as evidence that they are physically related to a common energy release at the Sun. The region of the solar energy release and the spacecraft are therefore considered as being magnetically connected, and the events are referred to as "well-connected" events.

In the literature the term "well-connected" is in general employed for solar activity that occurs in some restricted range of heliolongitudes around the nominal footpoint at the Sun of the Parker spiral through the observing point, the Earth or a spacecraft. Since the Parker spiral is an average description of the interplanetary magnetic field, this definition may not be adequate in each individual case, notably when the interplanetary magnetic field is perturbed by coronal mass ejections (Richardson \& Cane 1996; Masson et al. 2012). In addition, even when the interplanetary magnetic field is adequately described by a Parker spiral, energetic particles may have access to a given field line from a broad range of heliolongitudes. This is the case on the one hand when the acceleration region is broad, for instance an extended shock front (Lee et al. 2012). On the other hand, the Parker spiral is rooted on the source surface of the solar wind, at some distance from the photosphere. The open magnetic field lines that connect an active region in the low corona to this footpoint may spread apart with increasing altitude and cover an extended range of heliolongitudes (Klein et al. 2008) In all these cases SEPs can reach the spacecraft along magnetic field lines from longitudes that would be characterized as being poorly-connected if the definition referred to the nominal Parker spiral. The direct comparison between the rise of particle intensities at a spacecraft and a signature of coronal activity gives physical meaning to the term "connection".

The UMASEP model for predicting well-connected events, called here WCP model, issues an SEP prediction if at least one of the correlations between the proton intensities and the soft X-ray flux is high, and if the associated X-ray burst is also strong. This approach has two limitations: on the one hand, the correlation between the rises of the X-ray emission and the SEP intensity must not be coincidental. This is a hypothesis, which is validated by the success of the forecasting procedure. On the other hand, the procedure works only when the solar activity is on the visible disk. SEP events may be observed at Earth even when the parent activity is behind the solar limb. This can be due to the interplanetary transport, which may carry SEPs across magnetic field lines (Dresing et al. 2014; Laitinen \& Dalla 2017), or to a direct magnetic connection. However, the peak intensity and therefore the space-weather relevance of events that are more than $10^{\circ}$ behind the west limb or more than $20^{\circ}$ east of central meridian decreases significantly, as shown for instance in Figure 12 of Richardson et al. (2014). Within the UMASEP scheme, such events can still be predicted by a different approach, called the "poorlyconnected" (PCP) model, which does not employ electromagnetic data. For this reason we do not consider this model any more in the following. The term "poorly connected" is misleading in those cases where parent activity behind the limb has a magnetic connection to the terrestrial observer. This has to be kept in mind when employing the conventional UMASEP nomenclature as described above.

The aforementioned scheme has been used to build several tools: UMASEP-10 (Núñez 2011), the first of these tools, predicts well- and poorly-connected SEP events $>10 \mathrm{MeV}$ from soft X-ray and proton fluxes; UMASEP-100 (Núñez 2015), a tool for predicting well-connected $>100 \mathrm{MeV}$ SEP events from soft X-ray and proton data; HESPERIA 
UMASEP-500 (Núñez et al. 2017, in preparation), a tool for predicting well-connected $>500 \mathrm{MeV}$ events from soft X-ray, proton and neutron monitor data. HESPERIA UMASEP$10 \mathrm{mw}$, the tool that is introduced in the present paper, is devised to predict SEP events with energies $>10 \mathrm{MeV}$ from microwave and proton data. Real-time UMASEP-10 forecasts are publicly available since 2010 in NASA's integrated Space Weather Analysis (iSWA) system, ${ }^{2}$ in the European Space Weather Portal, ${ }^{3}$ as well as in the University of Malaga's space-weather portal. ${ }^{4}$ UMASEP-10 was also included as a module in the European Space Agency's SEPsFLAREs system (García-Rigo et al. 2016). Section 2.2 describes the UMASEP scheme, and Section 2.4 the adaptation of this scheme to build the tool UMASEP-10mw using microwave data.

\subsection{UMASEP-10: the UMASEP scheme based on soft X-ray data}

The magnetic connectivity estimation of the well-connected prediction (WCP) model is based on the strength of the correlation between the time derivatives of the soft X-ray flux and the differential proton flux in at least one of the channels between 9 and $500 \mathrm{MeV}$ measured by all available GOES satellites, as Figure 1a illustrates. A persistent high correlation is considered as a signature that particles are escaping along magnetic field lines to the observer. For the case of UMASEP-10, a forecast is triggered when a magnetic connection is detected and the associated X-ray flux peak is greater than $4 \times 10^{-6} \mathrm{~W} \mathrm{~m}^{-2}$ ( $>\mathrm{C} 4$ flares). The best results are obtained when evaluating the correlation between the time derivatives of soft X-ray and proton fluxes at time $t$, both normalized to 1 , where $t$ is the time stamp in 5 -min integrated data.

This approach tries to identify potential cause-consequence pairs of positive time derivatives. A positive time derivative of the soft X-ray flux is analysed only if it exceeds a threshold $h$ in the interval from time step $t-1$ to $t$. This threshold is set to eliminate triggering by background fluctuations. A pair is discarded if the time between the soft X-ray increase and the consequential proton increase is shorter than two time steps, i.e. $10 \mathrm{~min}$. This interval accounts for the fact that it takes the protons a longer time to travel to the spacecraft than the photons. The numerical value is adjusted empirically. Because there are several ways to pair X-ray rises to differential proton flux rises, the approach collects all possible combinations of consecutive cause-consequence pairs. The set of possible cause-consequence pairs belonging to an observed significant increase of the soft X-ray flux is called a CCsequence.

To estimate the correlation, a fluctuation similarity is calculated. Each CCsequence has a set of possible causeconsequence pairs. Let a given CC-pair be labelled $(i, j)$, where index $i$ refers to the time of the soft X-ray measurement, index $j$ to that of the proton measurement. With each such pair we can associate a time difference $\Delta t_{i j}=\operatorname{time}(i)-\operatorname{time}(j)$ and an intensity difference of the protons $\Delta J_{i j}=J_{\mathrm{p}}(i)-J_{\mathrm{p}}(j)$. A cause-effect pattern between two measurements $i$ and $j$ is identified when a sequence of pairs has very similar time differences and intensity differences, and when this situation

\footnotetext{
${ }^{2}$ http://iswa.ccmc.gsfc.nasa.gov/IswaSystemWebApp/index.jsp?i_1= 1411_1=40 t_1=270 w_1=600 h_1 $=500$

${ }^{3} \mathrm{http}: / /$ www.spaceweather.eu/forecast/uma_sep

${ }^{4} \mathrm{http} / / /$ spaceweather.uma.es/forecastpanel.htm
}

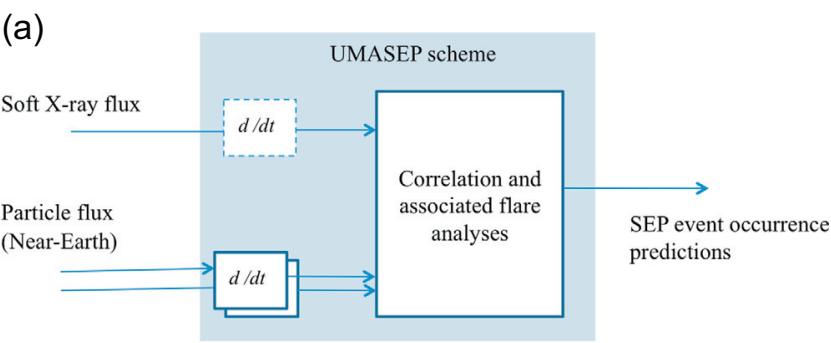

(b)

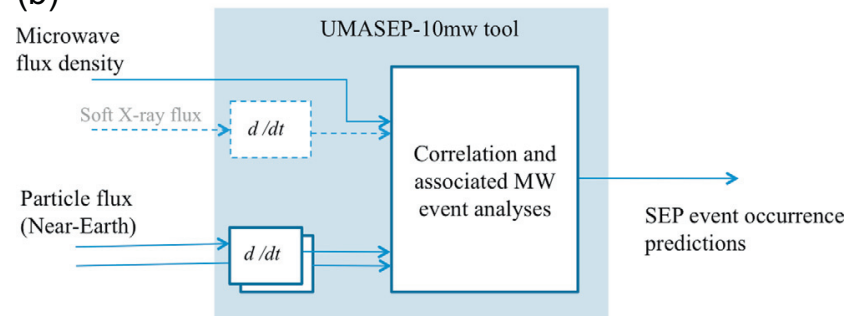

Fig. 1. Schematic of the correlation process of the Well-Connected SEP forecasting module of the UMASEP scheme. (a) UMASEP-10, which correlates the time derivative of soft X-ray flux with the time derivative of the differential proton fluxes in different energy channels observed by the GOES spacecraft $(9-500 \mathrm{MeV})$. (b) UMASEP-10mw, which uses the microwave flux density instead of the soft X-ray derivative.

persists over a minimum duration $d$. To measure the similarity function $s_{i j}$, where $i$ and $j$ are the analysed subsequences, we used an ad hoc formula:

$$
s_{i j}=w_{t} \frac{\mu_{t}+\epsilon}{\mu_{t}+\sigma_{t}+\epsilon}+w_{J} \frac{\mu_{J}+\epsilon}{\mu_{J}+\sigma_{J}+\epsilon},
$$

where $w_{t}$ and $w_{J}$ are weights of the similarity in terms of temporal and intensity differences, respectively; $\mu_{t}$ and $\sigma_{t}$ are the average and the standard deviation of the time differences $\Delta t_{i j}$ of the pairs within a CCsequence; $\mu_{J}$ and $\sigma_{J}$ are the average and the standard deviation of the intensity differences of the pairs within a CCsequence; $\epsilon$ is a very small value used to avoid possible divisions by 0 . All these parameters were manually tuned to augment the probability of detection (POD) and reduce the false-alarm ratio (FAR). The WCP model calculates $s_{i j}$ for every differential proton channel $j$. Then it selects the highest $s_{i j}$, called $s_{\max }$ in the following, which is processed as follows:

- If the fluctuation similarity $s_{\max }$ is lower than a threshold $m$, it is considered that particles are not accelerated during the eruptive event, or else that there is no magnetic connection to the Earth.

- If the fluctuation similarity $s_{\max }$ is greater than or equal to the fluctuation-similarity threshold $m$, two conclusions are issued: there is a magnetic connection with normalized strength $s_{\max }$, and the average of the temporal distances between the causes and consequences within CCsequence is the estimated supplementary travel time of protons, as compared to photons, from the Sun to 1 AU. The associated flare may be identified in the information within CCsequence. The highest original (X-ray) flux of the corresponding causative fluctuations in pairs within CCsequence corresponds to the peak of the associated flare. If the peak of the associated flare is greater than a certain X-ray flux threshold $f$, then a preliminary 
well-connected SEP forecast is sent to the Analysis and Inference Module, including the time and X-ray peak flux of the associated flare.

The UMASEP-10 tool uses this scheme with soft X-ray and proton fluxes for predicting protons above $10 \mathrm{MeV}$. As mentioned earlier, in addition to forecasting well-connected events, UMASEP-10 also has a poorly-connected event prediction (PCP) model. The performance of the combined UMASEP-10 WCP and PCP models on GOES soft X-ray and proton data, updated for version 1.3 (Núñez 2015), obtained a POD of $88.6 \%$ and a FAR of $23.24 \%$, and an average warning time of $3 \mathrm{~h} 58 \mathrm{~min}$, for the period of January 1994-September 2013.

For every predicted well-connected SEP event, the UMASEP-10 tool also predicts the integral proton flux that will be attained $7 \mathrm{hr}$ after the time of the prediction. The procedure is summarized as follows: the $>10 \mathrm{MeV}$ integral proton flux $7 \mathrm{hr}$ after the time of the prediction, called $I_{7 \mathrm{~h}}$, is calculated as:

$$
I_{7 \mathrm{~h}}=A\left(F \times 10^{S_{\max }}\right)+B,
$$

where $A$ and $B$ are linear regression factors that were empirically found with observed $I_{7 \mathrm{~h}}$ values in historical well-connected SEP events that took place in solar cycles 22 and $23, s_{\max }$ is the maximum similarity value calculated from the recent soft X-ray and proton fluxes (see above), and $F$ is the time-integral of the recent soft X-ray flux calculated from near the flare onset to the flare peak. For more information about the aforementioned formula, see Núñez (2011).

\subsection{Non-thermal microwave bursts and the Neupert effect}

Radio emission at microwave frequencies has contributions from three processes, which may or may not occur together during a given event: gyrosynchrotron emission from nonthermal electrons at energies between about $100 \mathrm{keV}$ and a few $\mathrm{MeV}$, thermal bremsstrahlung and coherent plasma emission from anisotropic non-thermal electron distributions, such as beams. Thermal bremsstrahlung emission is usually rather weak $(<100 \mathrm{sfu})^{5}$ and has a spectrum that rises at frequencies around $5 \mathrm{GHz}$, to a flat peak at frequencies above about $9 \mathrm{GHz}$. The peak frequency varies from event to event. Plasma emission is most clearly seen at the lower frequencies $(\leq 3 \mathrm{GHz})$ and usually has a very rapidly varying time profile.

Empirically it is known that the most intense microwave emission usually occurs during the rise phase of the soft $\mathrm{X}$-ray burst, and that its light curve mimics the time derivative of the soft X-ray flux (Neupert 1968) - the so-called Neupert effect. The hard X-ray light curve has a similar relationship with the soft X-ray derivative (Dennis \& Zarro 1993; Holman et al. 2011). This points to a common time evolution of the energy release that goes to the electron acceleration on the one hand and to the heating of the plasma during the related flare on the other. Since the UMASEP scheme uses the derivative of the soft X-ray time profile and the proton profile to identify a magnetic connection to a solar particle source, one should be able to replace the calculated soft X-ray derivative by the observed microwave time profile. To do this, one must make sure that the used time profile is due to the

\footnotetext{
${ }^{5} 1 \mathrm{sfu}$ (solar flux unit) $=10^{-22} \mathrm{~W} \mathrm{~m}^{-2} \mathrm{~Hz}^{-1}$
}
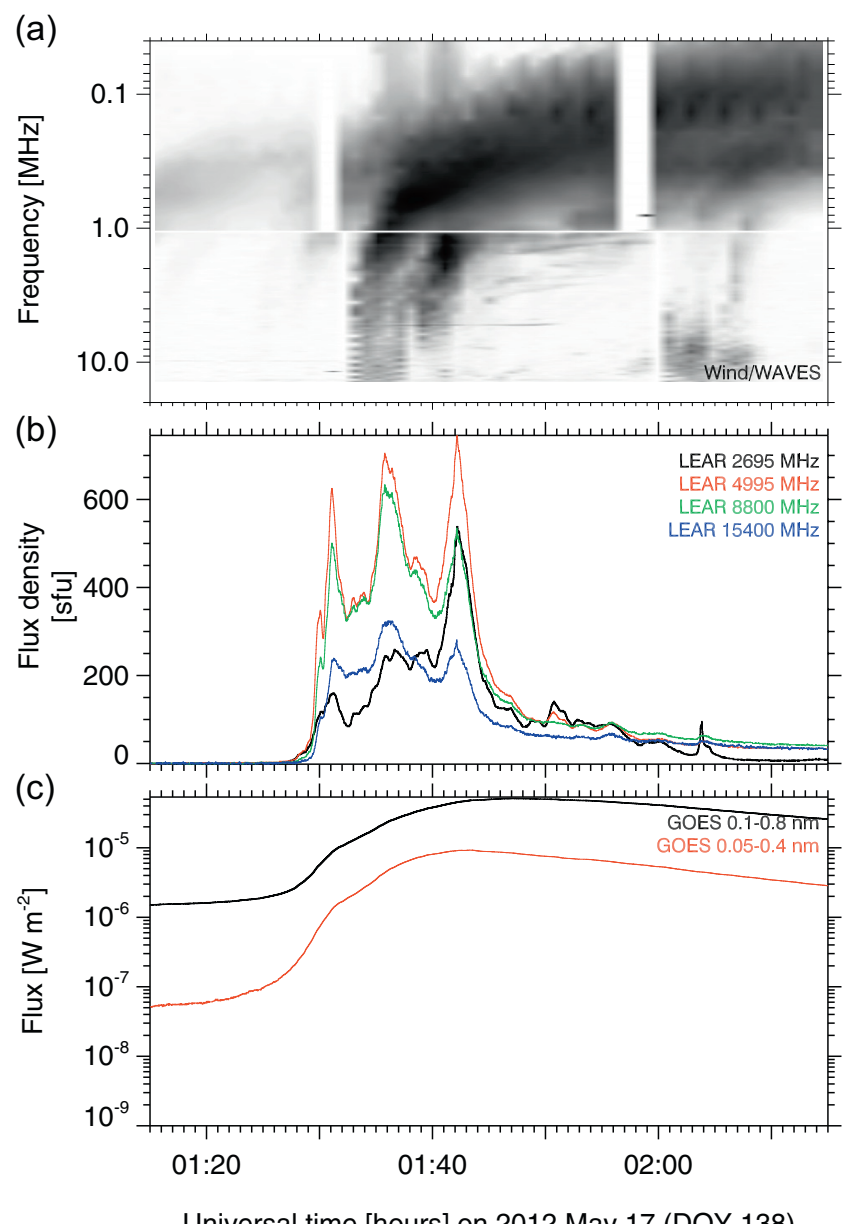

Universal time [hours] on 2012 May 17 (DOY 138)

Fig. 2. Time history of the soft X-ray (c), microwave (b) and decametre-to-kilometre-wave radio emission (a) associated with the SEP event on 2012 May 17. The grey-scale plot in (a) shows a dynamic spectrum, with dark shading showing bright emission.

gyrosynchrotron emission of mildly relativistic electrons. The Neupert effect breaks down when the microwave emission is dominated by thermal bremsstrahlung.

Multi-wavelength observations of a solar soft X-ray and radio burst are displayed in Figure 2. The emissions accompany the solar origin of a large SEP event, which was also detected at ground level by neutron monitors. The rise of the soft X-ray emission (Fig. 2c) comprises two bursts, each with a microwave counterpart shown in the Figure $2 b$. The microwave emission is pronounced in the rise phase of the X-ray burst, consistent with the Neupert effect. The emission has a broadband component, with similar peaks being seen at 4.995 (red curve), 8.8 (green) and $15.4 \mathrm{GHz}$ (blue). This is a typical signature of gyrosynchrotron emission from mildly relativistic electrons. At each frequency between 4.995 and $15.4 \mathrm{GHz}$ a prolonged, gradually decreasing weak emission is seen in the decay phase, say after 01:45 UT. This slowly evolving emission with flux density below $100 \mathrm{sfu}$ is the typical signature of thermal bremsstrahlung. It is much weaker than the non-thermal gyrosynchrotron emission, which usually dominates during the impulsive flare phase. The time profile at $2.695 \mathrm{GHz}$ (black curve) has similarities with the higher frequencies, in that it shows the same overall peaks, but with different amplitudes. This reveals the changing gyrosynchrotron spectrum in the course of the event. The decay of 
the time profile does not show the thermal bremsstrahlung signature, which is optically thick at $2.695 \mathrm{GHz}$. But there are smaller bursts, which do not show up at 8.8 and $15.4 \mathrm{GHz}$. They may be due to plasma emission. Plasma emission may also dominate the gyrosynchrotron emission in certain events at frequencies up to some GHz. It does not necessarily have the relationship with soft X-rays described by the Neupert effect.

The dynamic spectrum in Figure 2a shows type III bursts from electron beams between the high corona, at a heliocentric distance of the source at $10 \mathrm{MHz}$ of about $3 \mathrm{R}_{\odot}$ (e.g. Mann et al. 1999), and $1 \mathrm{AU}$ near $20 \mathrm{kHz}$. The typical drift towards lower frequencies shows the beams are propagating outward. Their appearance at the time of the impulsive phase of the flare, when the microwave emission is bright, shows that electrons accelerated in the flaring active region find access to the high corona and interplanetary space. This makes it likely that protons accelerated during the impulsive phase also escape to the interplanetary space.

\subsection{The UMASEP-10mw tool}

Based on the UMASEP scheme, illustrated in Figure1a, the UMASEP-10mw tool was developed. In order to construct the tool UMASEP-10mw for predicting $>10 \mathrm{MeV}$ SEP events using microwave data, the time derivative of the soft X-rays was replaced by the microwave flux density, as illustrated in Figure 1b. The UMASEP thresholds were re-calibrated. The tool UMASEP-10mw has been developed to be used for calculating the correlation between the solar microwave flux densities at 4.995 and $8.8 \mathrm{GHz}$, which are monitored by patrol instruments (see Sect. 3), and the time derivatives of the nearearth differential proton fluxes measured in different energy channels (i.e. using the GOES satellites). The rest of this section describes in detail how the UMASEP scheme was adjusted to properly use microwave data for predicting $>10 \mathrm{MeV}$ SEP events; Section 3 presents the preliminary results of this tool. For brevity, and since the emission is intrinsically broadband, we refer to the two microwave frequencies as 5 and $9 \mathrm{GHz}$ instead of 4.995 and $8.8 \mathrm{GHz}$.

The first calibration of UMASEP using microwave data was done using a set of thresholds that was very similar to that using soft X-ray data; however, the results in terms of probability of detection (POD) and false-alarm ratio (FAR) were not satisfactory. We found that the use of similar threshold values as UMASEP-10 led to a poor performance mainly because there are important differences between the time derivatives of soft X-rays and the microwave flux density in terms of candidate events, that is events where the time history has a positive slope during several successive time intervals. Because of the many fluctuations of the thermal soft X-ray emission of the Sun we had to impose a threshold $f$ of the peak X-ray flux to be considered in UMASEP-10 when triggering an SEP event prediction. Microwave data are more robust, in the sense that a conspicuous microwave burst usually takes place when electrons are accelerated to near relativistic energies. This occurs much less often than a thermal X-ray burst, such that we did not need to impose a threshold $f$ within UMASEP-10mw.

We searched for an optimal configuration of the parameter $l$, thresholds $h, m, d$, and the weights $w_{t}$ and $w_{J}$ (factors of the similarity function) so as to increase the POD and reduce the FAR in the forecast of well-connected SEP events. By default, a general forecasting performance measure was needed to find the optimal configuration. We used a combination of precision, i.e. 1 - FAR, and recall, i.e. POD, with the corresponding weights: $\quad w(1-$ FAR $) \times(1-$ FAR $)+w_{\text {POD }} \times$ POD $\quad($ Davis $\&$ Goadrich 2006). With these types of multi-objective problems, designers usually give more weight to one objective than to the other. We decided to give equal importance to POD and 1 - FAR; therefore, the weights are 0.5 . To find a highly effective configuration of weights (not necessarily the best one), parameters and thresholds, we used a multi-resolution optimization. That is, we first searched the two optimal threshold configurations using low-resolution steps. For every configuration found, we applied a new search by using higher-resolution steps in the neighbourhoods of the solutions found in the previous step. The width of the new range for every threshold/ weight (to be optimized using higher-resolution steps) was a tenth of the original low-resolution width. We repeated the process until the highest general forecasting performance was reached over the studied time interval from December 2011 to December 2012.

\section{A test run of UMASEP using microwave data}

\subsection{A composite microwave time profile over 13 months from RSTN data}

The Radio Solar Telescope Network (RSTN) of the US Air Force provides continuous time series of whole-Sun flux densities at eight frequencies $(0.245,0.410,0.610,1.415$, $2.695,4.995,8.8,15.4 \mathrm{GHz})$ with $1 \mathrm{~s}$ time resolution. It comprises four different observatories located in western Australia (Learmonth), Italy (San Vito), Massachusetts (Sagamore Hill) and Hawaii (Palehua). The data are available via the National Geophysical Data Center (NGDC). ${ }^{6}$ Data from the Nobeyama Radio Polarimeters ${ }^{7}$ (NoRP; Torii et al. 1979; Nakajima et al. 1985), operated by the National Astronomical Observatory of Japan, were used for checking purposes and to replace RSTN/Learmonth when necessary.

There is no generally referenced publication on RSTN single-frequency patrol observations. A paper by Kennewell from June 2008 is available on the web. ${ }^{8}$ The following information is drawn from this publication. The equipment is the same at the four stations. The observations at frequencies between 1.415 and $8.8 \mathrm{GHz}$ on the one hand, $15.4 \mathrm{GHz}$ on the other, are carried out with two parabolic antennas of diameters $2.4 \mathrm{~m}$ and $1 \mathrm{~m}$, respectively. They track the Sun from sunrise to sunset. The observing periods of the four stations overlap. This overlap can be used for the intercalibration.

Kennewell notes that power supply fluctuations, pointing errors and occasional drive problems are such that the tracking may have to be corrected manually. These corrections are carried out when the operator notes that the output signal is lower than expected. The corrections are hence delayed with respect to the occurrence of the problem, which leaves traces in the data such as drifts and sudden changes of the flux density. We developed several simple procedures for a semiautomated correction of some of the problems:

- Observing intervals in the early morning and late afternoon are cut out in order to avoid periods with bad pointing.

\footnotetext{
${ }^{6} \mathrm{http} / / /$ www.ngdc.noaa.gov/stp/space-weather/solar-data/solarfeatures/solar-radio/rstn-1-second/

${ }^{7} \mathrm{http}: / /$ solar.nro.nao.ac.jp/norp/html/event/

${ }^{8}$ www.deepsouthernskies.org/LSO/RSTN.pdf
} 
(a)

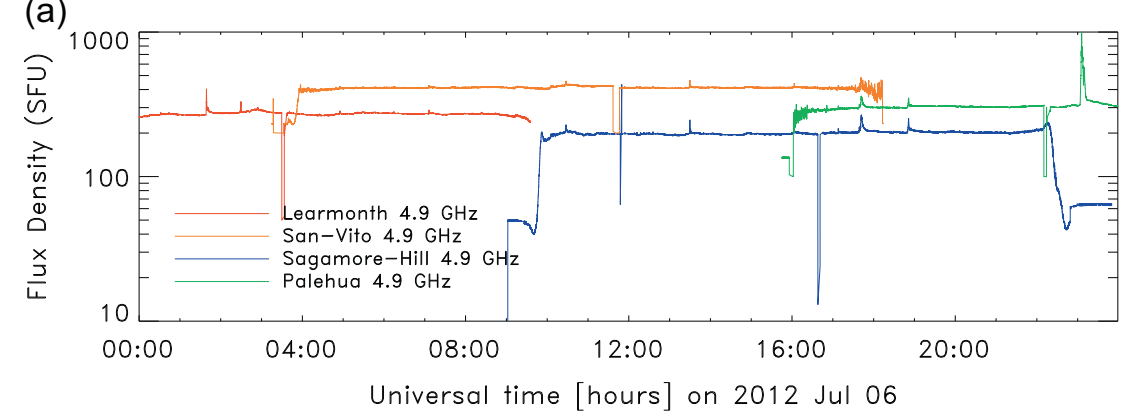

(b)

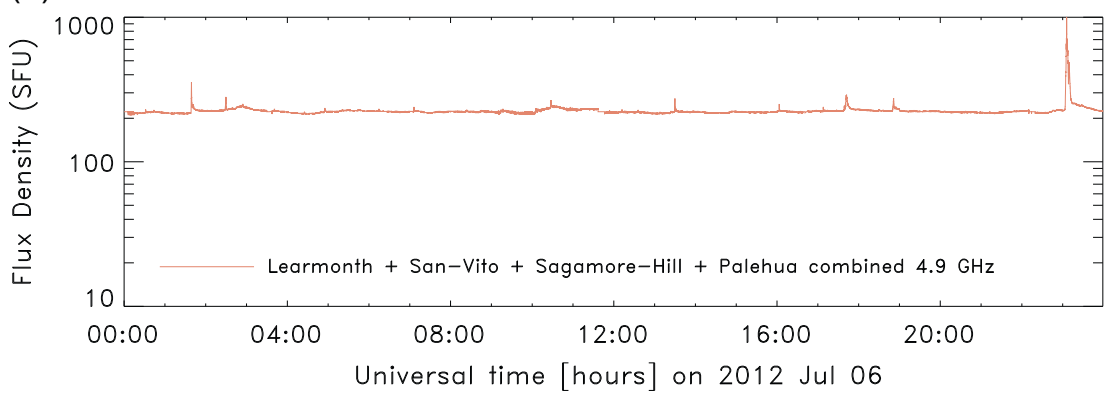

Fig. 3. Example of microwave data for a sample 24-hr interval. (a) The flux density observed by the four RSTN stations at 4.9 GHz. Spikes, discontinuities and background are corrected in the combined flux density (b).

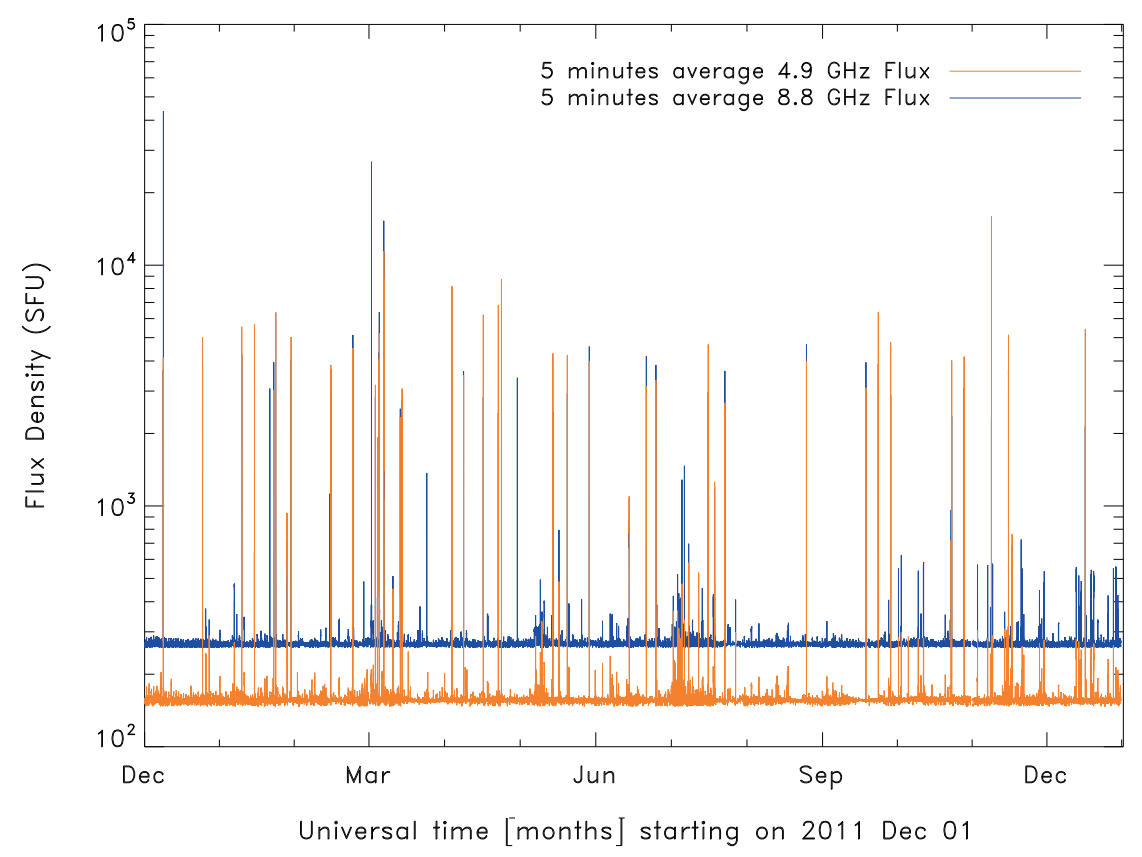

Fig. 4. The combined time history of the microwave flux density at two frequencies during the 13 months from 2011 December 01 to 2012 December 31, constructed from observations of the four RSTN stations. The flux density is averaged over 5 min, the background is removed for each instrument and an average backgound over the 13-month period is added.

- Isolated spikes are identified by a comparison of the flux density level with adjacent time intervals and cut out. The spikes are replaced by an average of the adjacent flux density values.

- At each frequency for each observing station a daily background is automatically determined in an iterative procedure: the average and standard deviation of the flux density are computed in the first run, and in an iterative procedure refined by omitting flux densities with absolute values that exceed the average by more than three standard deviations.

- The average of the background values of the four observing stations is then added to the background-subtracted flux densities of the individual stations. The background 
(a)

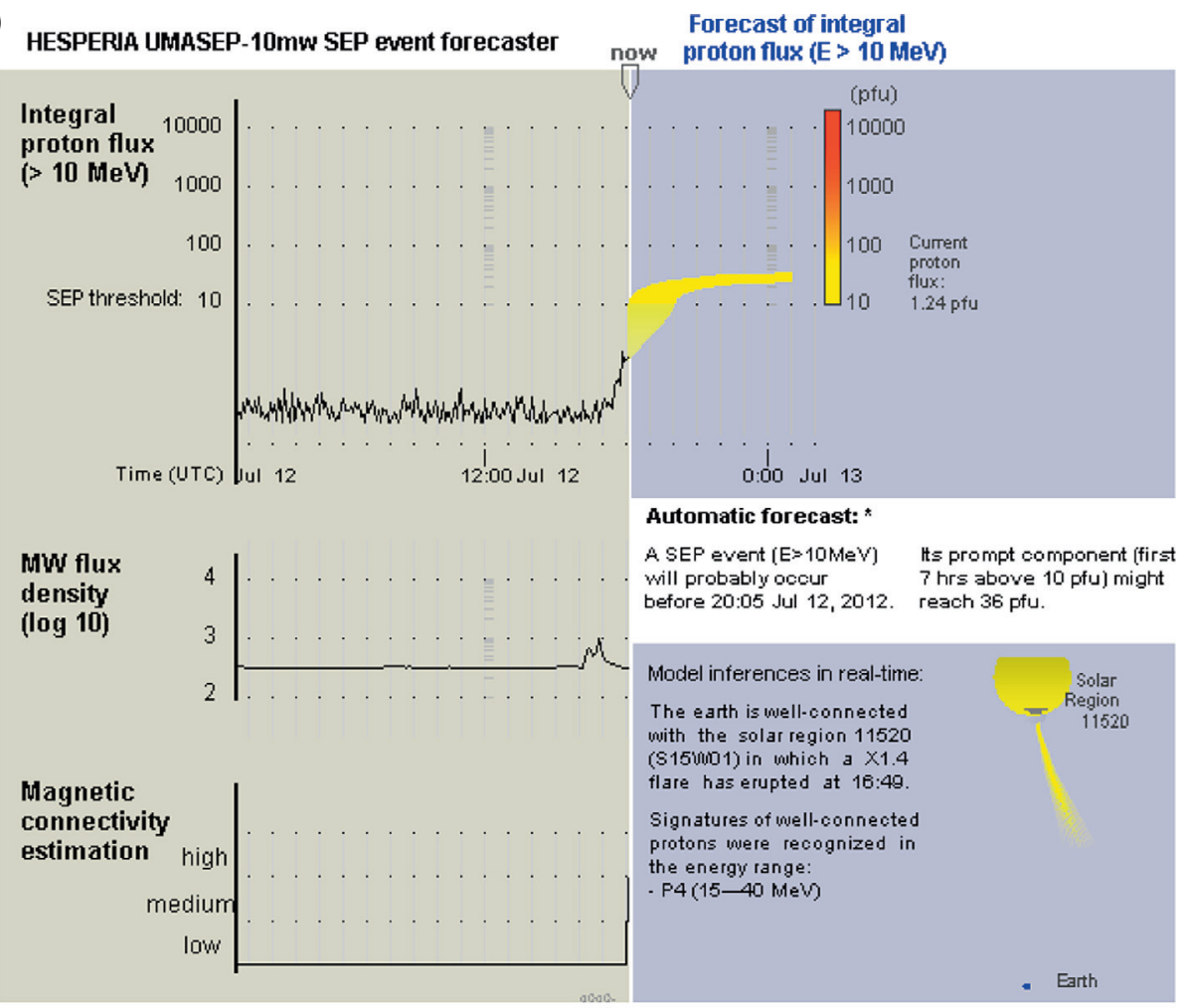

(b) HESPERIA UMASEP-10mw SEP event forecaster

Forecast of integral

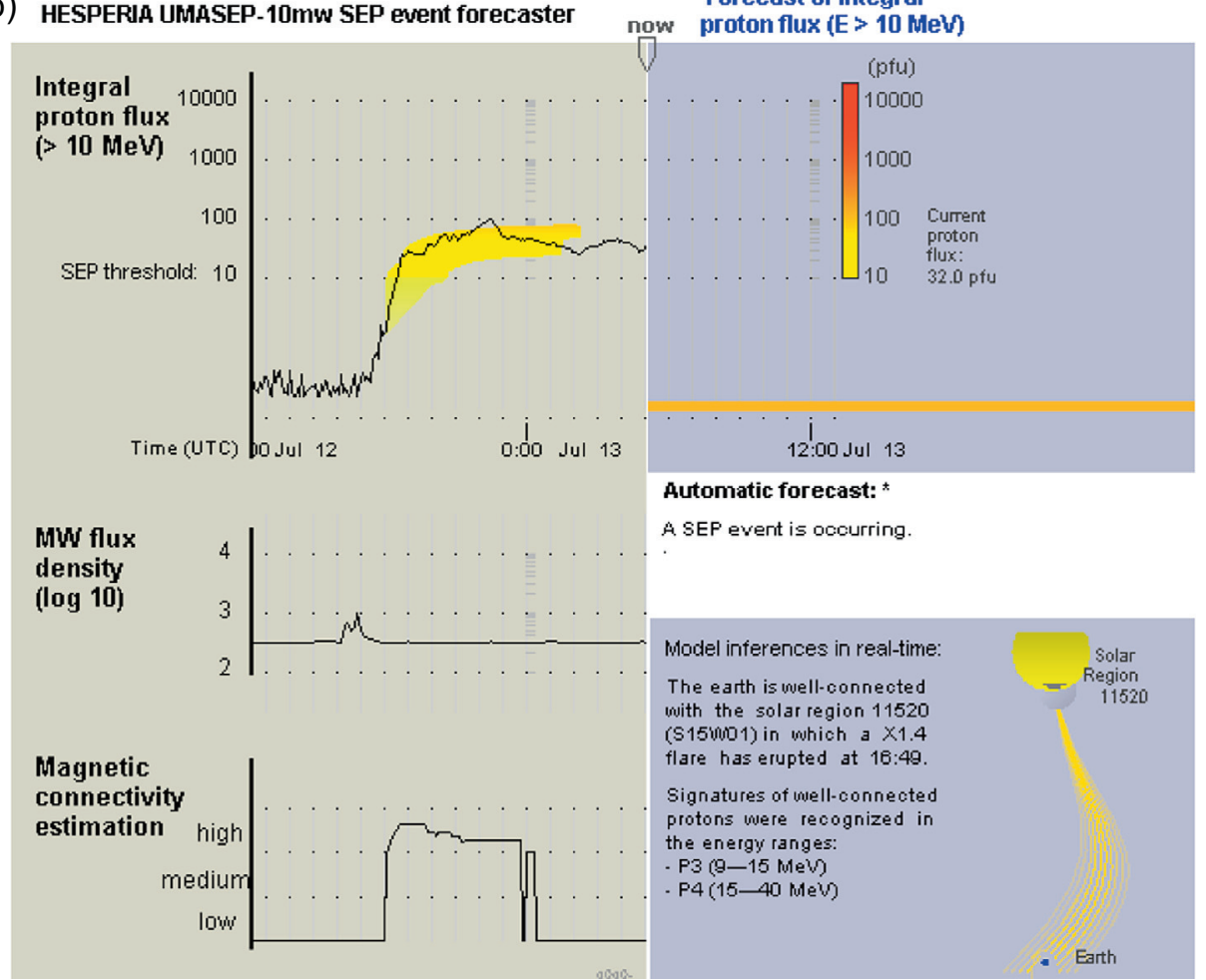

Fig. 5. Two UMASEP-10mw outputs after processing microwave data at $5 \mathrm{GHz}$ from 2012 July 12 and GOES proton fluxes of $>10 \mathrm{MeV}$ energies. (a) The prediction at 18:05. (b) The subsequent evolution of the $>10 \mathrm{MeV}$ integral proton flux. The yellow/orange band in the proton intensity plots gives the predicted range, with the colour scale shown by the vertical bar.

procedure removes discontinuities at the transition between different stations, but only as long as the individual background levels are constant.
- The daily records constructed in this way are then pasted together to build a long time series, up to 13 months. A uniform average background is added at each frequency, 
and smaller flux densities are set to the background value. This is done to avoid data gaps especially during calibration periods around local noon, when the antenna is pointed away from the Sun during several minutes. Finally, 5-min integration further smoothes out short-term irregularities that remain after the data cleaning procedure.

Figure 3 shows a sample 24-hr interval. In Figure 3 a the original data are plotted for the four RSTN stations, while Figure $3 \mathrm{~b}$ shows the corrected combined data after the semiautomatic procedure. Dips of the light curves in Figure 3a near the centres of the observing intervals are due to the abovementioned calibration periods. The selected observing time for each RSTN station varies depending on the period of the year. The time intervals are typically 00-08 UT for Learmonth, 08-14 UT for San Vito, 14-19 UT for Sagamore Hill and 19-24 UT for Palehua. For the period 2012 March 01-07, measurements from Learmonth were not available at $8.8 \mathrm{GHz}$, while from 2012 July 10-30, no Learmonth observations were available at all. The Learmonth data at $4.9 \mathrm{GHz}$ were replaced by Nobeyama measurements at $3.75 \mathrm{GHz}$, those at $8.8 \mathrm{GHz}$ by Nobeyama observations at $9.4 \mathrm{GHz}$.

Figure 4 shows the resulting flux density calculated for the 13-month interval from December 2011 to December 2012. At both frequencies numerous bursts are seen. The two light curves are used in the following to replace the first derivative of the soft X-rays in the UMASEP-10mw test.

\subsection{Illustration of an UMASEP-10mw forecast}

We illustrate the forecast of the UMASEP-10mw tool using microwave data at $5 \mathrm{GHz}$ for predicting the $>10 \mathrm{MeV}$ SEP event. We used independently the forecasting tools working exclusively with the soft X-ray derivative and exclusively with the microwave flux density, and compared their results. Figure 5 shows the forecast graphical output that an operator would have seen if the UMASEP-10mw tool had processed real-time microwave data on 2012 July 12 . This figure also shows the inferences about the associated flare, heliolongitude and active region.

Figure 5a displays the prediction before the SEP event and Figure $5 \mathrm{~b}$ the forecast image several hours after the start time. The upper time series in both images shows the observed integral proton flux with energies greater than $10 \mathrm{MeV}$. The current flux is indicated below the label "now" at each image. To the right of this label, the forecast integral proton flux is presented. The yellow/orange-coloured band indicates the expected evolution of the integral proton flux derived from the prediction of the proton flux $I_{7 \mathrm{~h}}$ as described in equation (2). The band shows the backward extrapolation of the range $I_{7 \mathrm{~h}} \pm 23 \%$ to the current time, using a function that increases as $t^{0.2}$, which was found to be a convenient average representation in past SEP events. In order to make the prediction of equation (2) work when microwave data are used as input, a simple linear relationship was determined between the derivative of the soft X-ray flux and the microwave flux density for the considered 13-month interval. The central curves in Figures $5 \mathrm{a}$ and $5 \mathrm{~b}$ display the microwave flux density time profile, and the lower time series shows the magnetic connectivity estimation (for more information, see Sect. 2.1) with the best-connected CME/flare process zone. When a forecast is issued, the graphical output also shows the details of these predictions and what the model infers about the situation. Figure 5 shows the prediction at 18:05 (2012 July 12). This forecast is that an event will start during the following $2 \mathrm{hr}$ and reach a peak intensity of $36 \mathrm{pfu}^{9}$ (see white section "Automatic forecast"). Below the forecast section, the system also presents the model inference section, which shows that the Earth is well connected with the solar region 11520 . The system also shows that the associated X1.4 flare took place at S15W01. As time passes, the integral proton flux also rises. At 18:35 UT, the flux exceeds the 10-pfu threshold, which indicates that a proton event is occurring. Note that the well-connected SEP event was successfully forecast $30 \mathrm{~min}$ earlier, when the enhancement of the integral proton flux was still weak (1.24 pfu).

\subsection{UMASEP-10mw forecasting using the microwave time profile}

In order to assess the performance of the UMASEP-10mw tool, it was run from December 2011 to December 2012. During this period, nine SEP events were considered as wellconnected events and four were considered as poorlyconnected events. The performance of this tool was assessed with the well-connected events only, because their predictions are directly associated to microwave emissions. Table 1 lists the SEP events with the obtained results. Column 1 gives the event start times, columns 2-4 the characteristics of the associated flare, columns 5-7 the warning time of the successful predictions and columns $8-10$ list the result of the predictions in terms of "hits" and "misses". Note that UMASEP-10mw (9 GHz) and UMASEP-10 have different results in the events on July 17 and September 28: the results of UMASEP-10mw were a "miss" and "hit", respectively, whilst the results of UMASEP-10 were "hit" and "miss". One event missed by the WCP model (2012 July 07) was successfully predicted by the PCP model, which is not supposed to predict such a well-connected event, and which is not applicable to UMASEP-10mw.

Taking into account the results in Table 1, Table 2 presents the forecast performance results in terms of POD, FAR and average warning time using only the Well-Connected forecasting model with microwave (5 and $9 \mathrm{GHz}$ ) or soft X-ray data. Probability of detection (POD) is the number of the predicted SEP events divided by that of the SEP events that actually occurred, i.e. nine events in the considered time interval. The false-alarm ratio (FAR) is the number of false predictions over the number of predictions. Seven predictions were triggered when microwaves were used, and eight with soft X-rays. An SEP event in the sense used here is an event where the proton intensity at energies above $10 \mathrm{MeV}$ exceeds $10 \mathrm{pfu}$. We note that the use of soft X-ray and microwave data produces the same POD. The most notable difference is that the use of microwave data does not yield any false alarm. The average warning time is slightly higher when microwave observations are used. The probabilities of detection used above are adequate to compare the performance of soft X-rays and microwaves within the UMASEP scheme, but overestimate the expected ones: SEP events originating behind the solar limb are undetectable to the UMASEP WCP scheme, because it uses electromagnetic observations from a terrestrial vantage point. This bias affects soft X-rays from GOES and radio observations from ground in the same way.

Regarding false alarms, it is interesting to note that on 2011 December 25 an M4 flare took place at 18:16. This

\footnotetext{
$91 \mathrm{pfu}=1 \mathrm{~cm}^{-2} \mathrm{~s}^{-1} \mathrm{sr}^{-1}$
} 
P. Zucca et al.: Microwave emission and forecasting of SEP occurrence

Table 1. Forecast results for each of the SEP events that occurred from November 2011 to December 2012 and were considered as wellconnected events, using soft X-ray (SXR) and microwave emission (5 and $9 \mathrm{GHz})$ as input to the UMASEP scheme.

\begin{tabular}{|c|c|c|c|c|c|c|c|c|c|}
\hline \multirow{2}{*}{$\begin{array}{c}\text { SEP } \\
\text { Start time }\end{array}$} & \multicolumn{3}{|c|}{ Flare } & \multicolumn{3}{|c|}{ Warning time (WCP model) ${ }^{(1)}$} & \multicolumn{3}{|c|}{ Result using WCP model $^{(1)}$} \\
\hline & Peak time & GOES class & Location & $5 \mathrm{GHz}(\mathrm{min})$ & $9 \mathrm{GHz}(\mathrm{min})$ & SXR (min) & $5 \mathrm{GHz}$ & $9 \mathrm{GHz}$ & SXR \\
\hline $\begin{array}{l}\text { 2012 Jan } 23 \\
05: 30\end{array}$ & $\begin{array}{l}\text { Jan } 23 \\
03: 59\end{array}$ & M8 & N28W36 & 50 & 50 & 45 & Hit & Hit & Hit \\
\hline $\begin{array}{l}2012 \text { Jan } 27 \\
\text { 19:05 }\end{array}$ & $\begin{array}{l}\text { Jan } 27 \\
18: 37\end{array}$ & $\mathrm{X} 1$ & N27W71 & 15 & 15 & 15 & Hit & Hit & Hit \\
\hline $\begin{array}{l}\text { 2012 Mar } 07 \\
05: 10\end{array}$ & $\begin{array}{l}\text { Mar } 07 \\
00: 24\end{array}$ & $\mathrm{X} 5$ & N17E15 & 25 & 25 & 70 & Hit & Hit & Hit \\
\hline $\begin{array}{l}\text { 2012 Mar } 13 \\
18: 10\end{array}$ & $\begin{array}{l}\text { Mar 13 } \\
17: 41\end{array}$ & M7 & N18W62 & 5 & 10 & 10 & Hit & Hit & Hit \\
\hline $\begin{array}{l}2012 \text { May } 17 \\
02: 10\end{array}$ & $\begin{array}{l}\text { May } 17 \\
01: 47\end{array}$ & M5 & N12W89 & 5 & 5 & 5 & Hit & Hit & Hit \\
\hline $\begin{array}{l}\text { 2012 Jul } 07 \\
04: 00\end{array}$ & $\begin{array}{l}\text { Jul } 06 \\
23: 08\end{array}$ & $\mathrm{X} 1$ & S18W50 & & & & Miss & Miss & $\operatorname{Miss}^{(2)}$ \\
\hline $\begin{array}{l}2012 \text { Jul } 12 \\
18: 35\end{array}$ & $\begin{array}{l}\text { Jul } 12 \\
17: 10\end{array}$ & $\mathrm{X} 1$ & S16W09 & 30 & 25 & 30 & Hit & Hit & Hit \\
\hline $\begin{array}{l}2012 \text { Jul } 17 \\
17: 15\end{array}$ & $\begin{array}{l}\text { Jul } 17 \\
17: 15\end{array}$ & M1 & S17W75 & & & 10 & Miss & Miss & Hit \\
\hline $\begin{array}{l}\text { 2012 Sep } 28 \\
03: 00\end{array}$ & $\begin{array}{l}\text { Sep } 27 \\
23: 57\end{array}$ & $\mathrm{C} 3$ & N08W41 & 85 & 85 & & Hit & Hit & Miss \\
\hline
\end{tabular}

(1) WCP is the abbreviation of "well-connected prediction".

(2) The UMASEP-10's WCP model did not predict this event. Due to its gradual start, this event was predicted by UMASEP-10's poorly-connected event model.

Table 2. Forecast performance results in terms of POD, FAR and average warning time of the UMASEP scheme (WCP model only) using microwave and soft X-ray (SXR) data from 2011 December 01 to 2012 December 31.

\begin{tabular}{lccc}
\hline \hline & \multicolumn{2}{c}{ UMASEP-10mw } & UMASEP-10 \\
\cline { 2 - 3 } & $(5 \mathrm{GHz})$ & $(9 \mathrm{GHz})$ & $(\mathrm{SXR})$ \\
\hline Probability of detection & $77.8 \%(7 / 9)$ & $77.8 \%(7 / 9)$ & $77.8 \%(7 / 9)$ \\
False-alarm ratio & $0 \%(0 / 7)$ & $0 \%(0 / 7)$ & $12.5 \%(1 / 8)$ \\
Average warning time & $30.7 \mathrm{~min}$ & $30.7 \mathrm{~min}$ & $26.4 \mathrm{~min}$ \\
\hline
\end{tabular}

western flare (S22W26) was associated with a small proton enhancement that did not exceed 10 pfu (i.e. no $>10 \mathrm{MeV}$ SEP event took place). At 23:25, UMASEP-10 detected a magnetic connection associated with the aforementioned flare, whose peak intensity was greater than the threshold $f$, the minimum X-ray peak flux (see Sect. 2.2), and, consequently, it issued a false alarm (see last column in Table 2). A microwave burst was also detected during this event, with a faint increase in both 5 and $9 \mathrm{GHz}$. But the flux densities did not exceed the threshold $h$, which suppresses triggering by background fluctuations. Therefore, UMASEP-10mw (successfully) did not issue any prediction. The aforementioned threshold $h$ in UMASEP-10mw was also useful to filter out all the faint microwave flux events artificially produced when the time profiles of two stations were joined. It is important to mention that during the first calibrations the threshold $h$ was wrongly set to a very low value; therefore, the number of false alarms of UMASEP$10 \mathrm{mw}$ was initially high. Once we set a proper threshold $h$ (i.e. to a value that is higher than the faint spurious microwave events, but lower than the real microwave events associated to SEP events), the number of false alarms abruptly decreased to 0 , without sacrificing successful predictions (see second and third columns of Table 2). This means that the threshold $h$ could be lowered if the microwave data quality were improved.

\section{Analysis of the results: missed events}

Table 1 shows that one of the two SEP events missed by UMASEP-10mw was also missed by UMASEP-10 (2012 July 07 ), while another one was successfully predicted (2012 July 17). The event 2012 September 28 was predicted by UMASEP-10mw, but missed by UMASEP-10. The reasons are examined in the following. The 2012 May 17 event, which was successfully predicted, but with a very short warning time, is also briefly discussed.

On 2012 July 07 a weak SEP event occurred with a peak intensity that barely exceeded the NOAA threshold of $10 \mathrm{pfu}$. Although the parent activity near $\mathrm{W} 50^{\circ}$ suggests a magnetic connection to the Earth, the particle intensity rose to its maximum slowly, during several hours, and in several steps, like during a poorly-connected SEP event. The UMASEP prediction web page is shown in Figure 6. When the well-connected prediction model was used, both UMASEP-10 and UMASEP-10mw failed to forecast the SEP event, although both the soft X-ray burst and the microwave burst were very clear. But the first derivatives of all differential proton intensities were noisy, and the correlation with either the soft X-ray derivative or the microwave flux density did not exceed the correlation threshold $s_{\max }$ of the UMASEP forecasting schemes. 
(a)
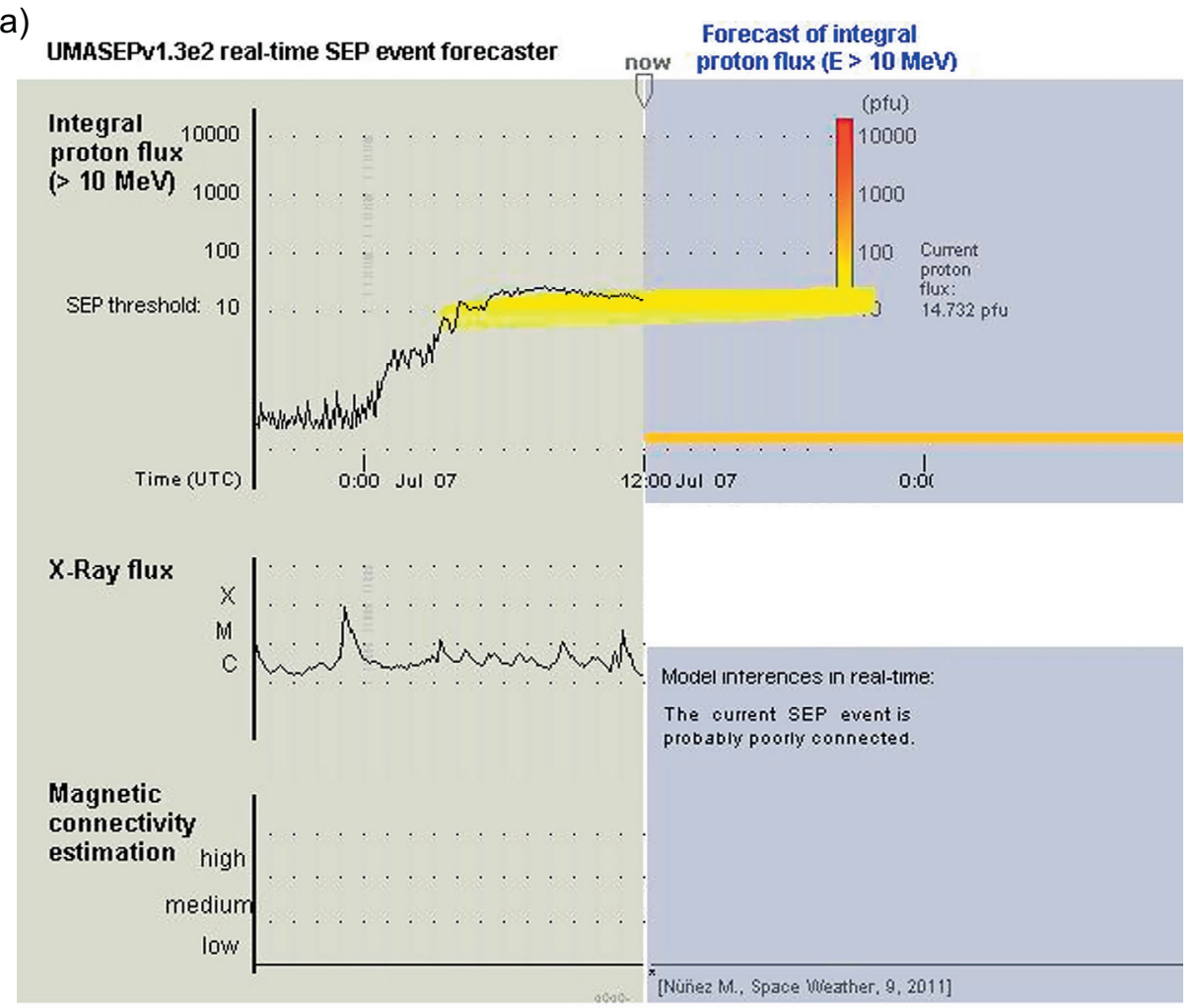

(b)
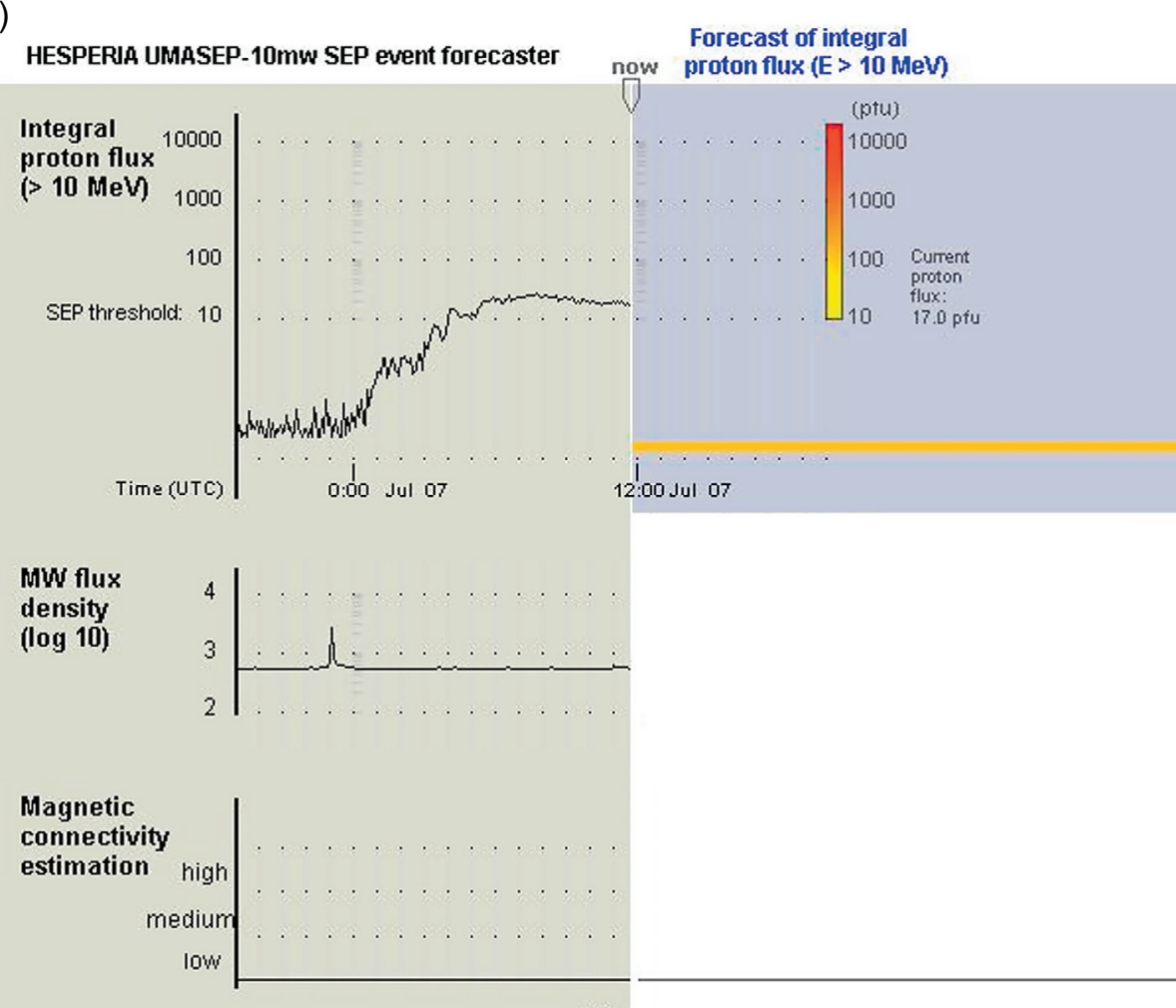

Fig. 6. UMASEP prediction web page for 2012 July 07: (a) and (b) show the SEP prediction using soft X-rays and microwaves, respectively The success of the prediction using soft X-rays is due to the poorly-connected prediction scheme. The well-connected prediction scheme failed to forecast the SEP event.

An SEP event without non-thermal microwave emission near 5 and $9 \mathrm{GHz}$ during a soft X-ray burst of importance M1.7 occurred on 2012 July 17-18. UMASEP-10 detected a magnetic connection, and the associated soft X-ray burst was strong enough to trigger an SEP forecast as shown in Figure 7a. The microwave burst had a slowly evolving time profile, with a 
(a)

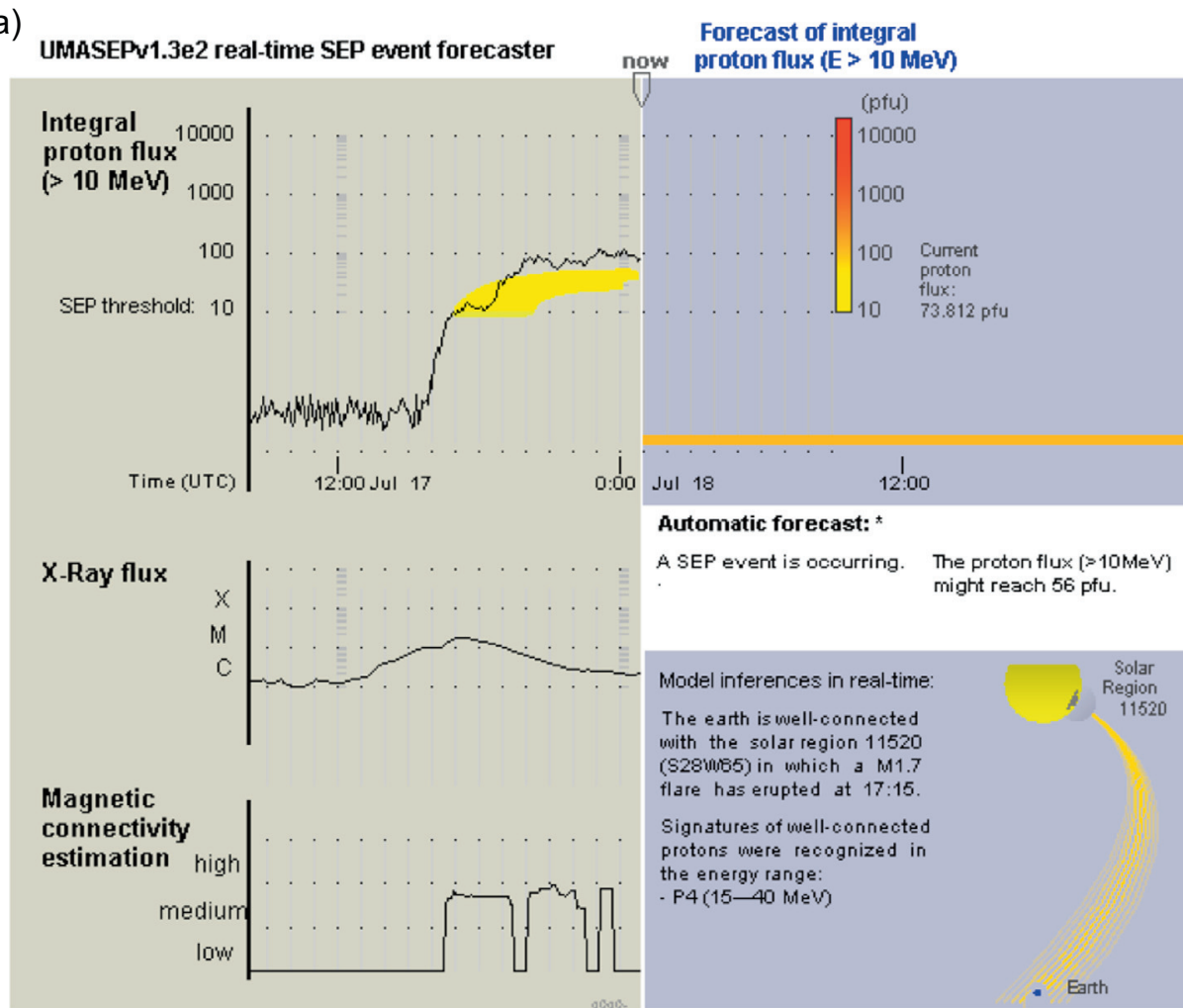

(b) HESPERIA UMASEP-10mw SEP event forecaster

Forecast of integral
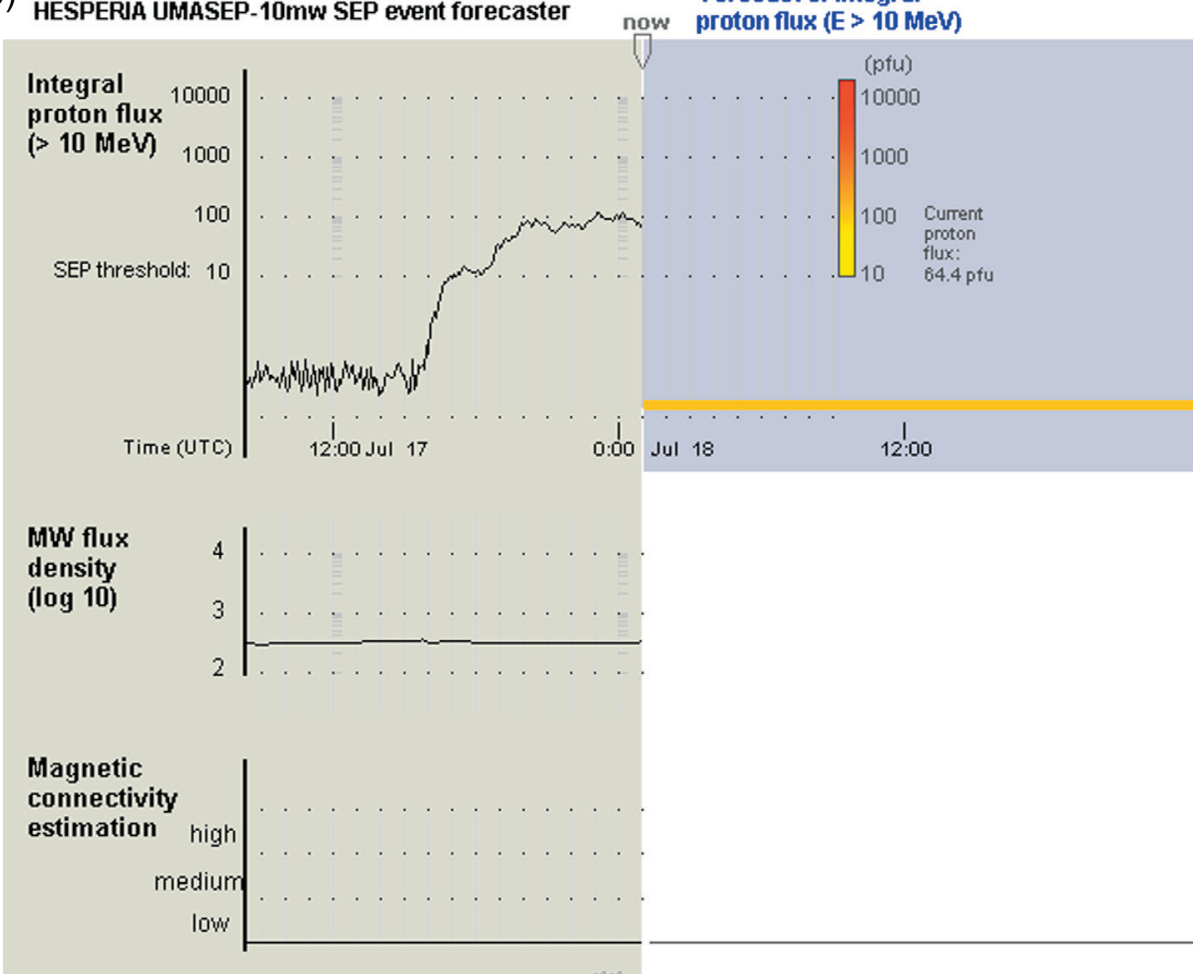

Fig. 7. UMASEP prediction web page for 2012 July 17: (a) and (b) show the SEP prediction using soft X-rays and microwaves, respectively.

rise from start to peak over about $40 \mathrm{~min}$, a flat high-frequency spectrum from 5 to $15 \mathrm{GHz}$, with a peak flux density around 40 sfu. This is typical of thermal bremsstrahlung. Because of the slow rise of the microwave time profile, only a rather weak correlation is found with the time derivative of the proton intensity profile. This correlation is below the similarity threshold $s_{\max }$, and no SEP forecast is issued by the UMASEP-10mw system, as shown in Figure 7b.

On 2012 September 28 an SEP event was preceded by a soft X-ray burst of class $\mathrm{C} 3$. This is below the UMASEP-10 
(a)

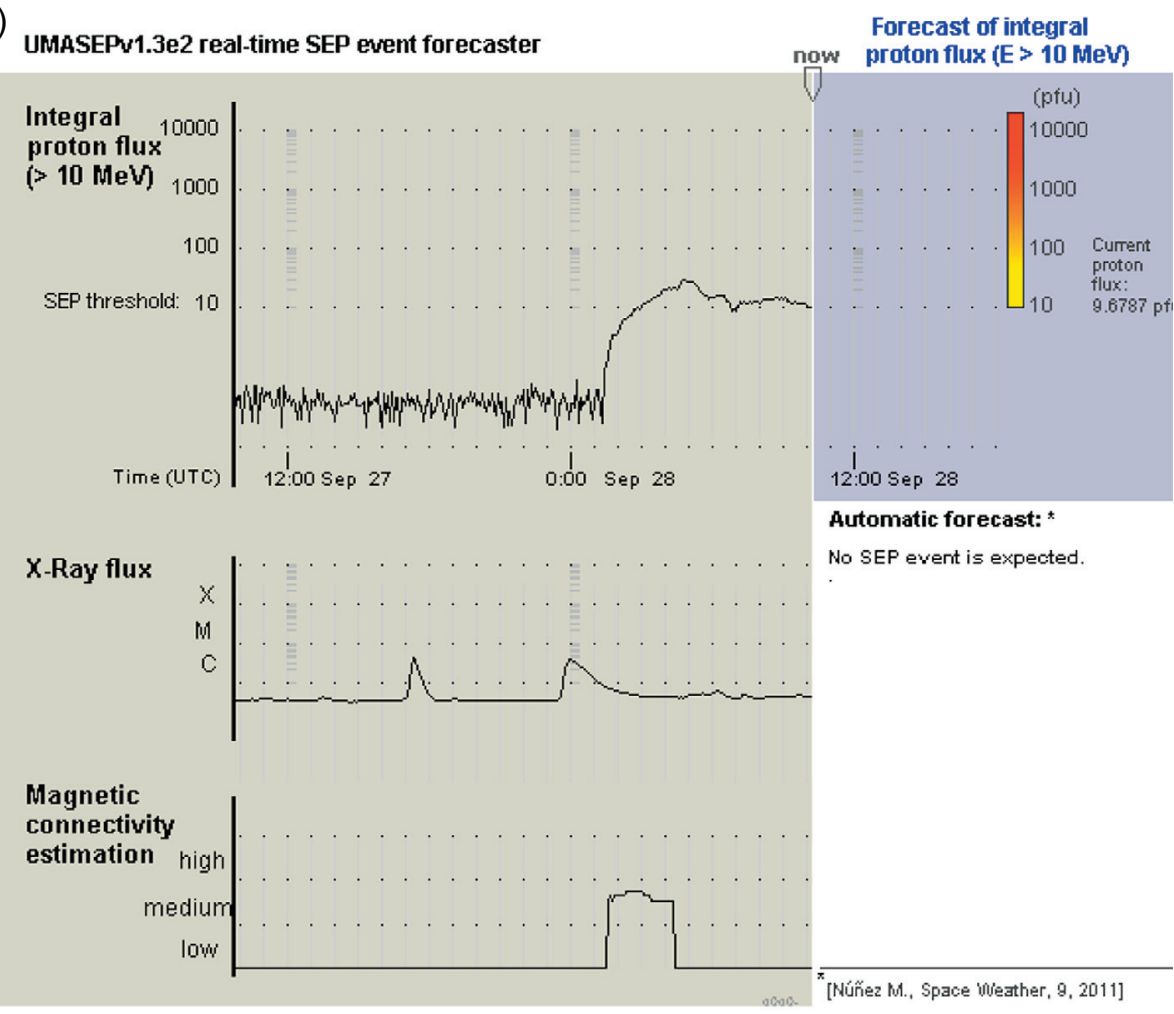

(b)

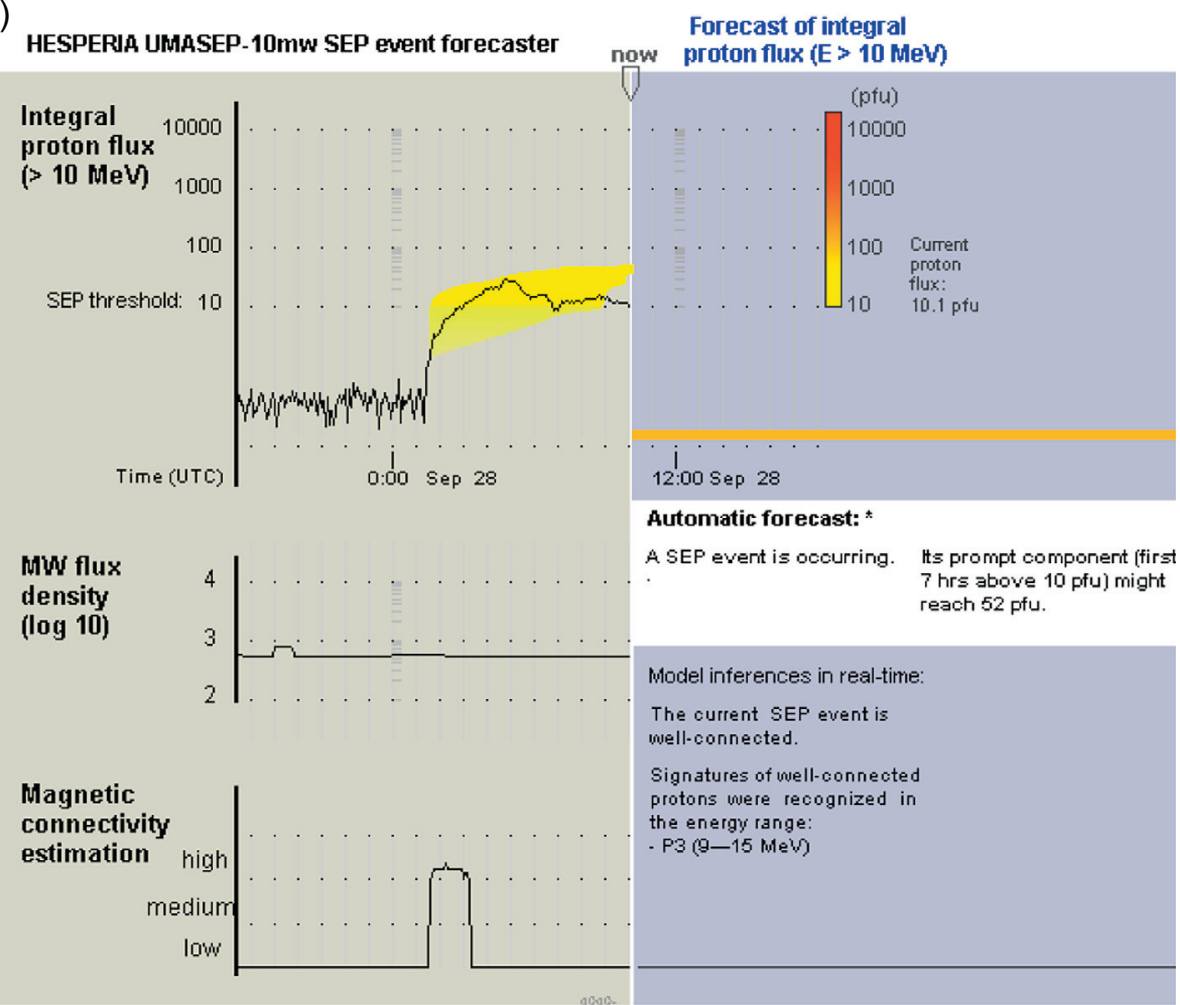

Fig. 8. UMASEP prediction web page for 2012 September 27: (a) and (b) show the SEP prediction using soft X-rays and microwaves, respectively.

threshold for event amplitudes (parameter $f$ ), and no SEP event was predicted based on the soft X-rays (Fig. 8a). The microwave emission at 5 and $9 \mathrm{GHz}$ was again thermal bremsstrahlung, with a rather low peak flux density (about 20 sfu at
$9 \mathrm{GHz}$ ), but a faster rise from background to peak (within $20 \mathrm{~min}$ ) than on 2012 July 17 . The thermal bremsstrahlung microwaves predicted the SEP event on September 28 (Fig. 8b), unlike the thermal soft X-rays. This success is due 
P. Zucca et al.: Microwave emission and forecasting of SEP occurrence

(a)

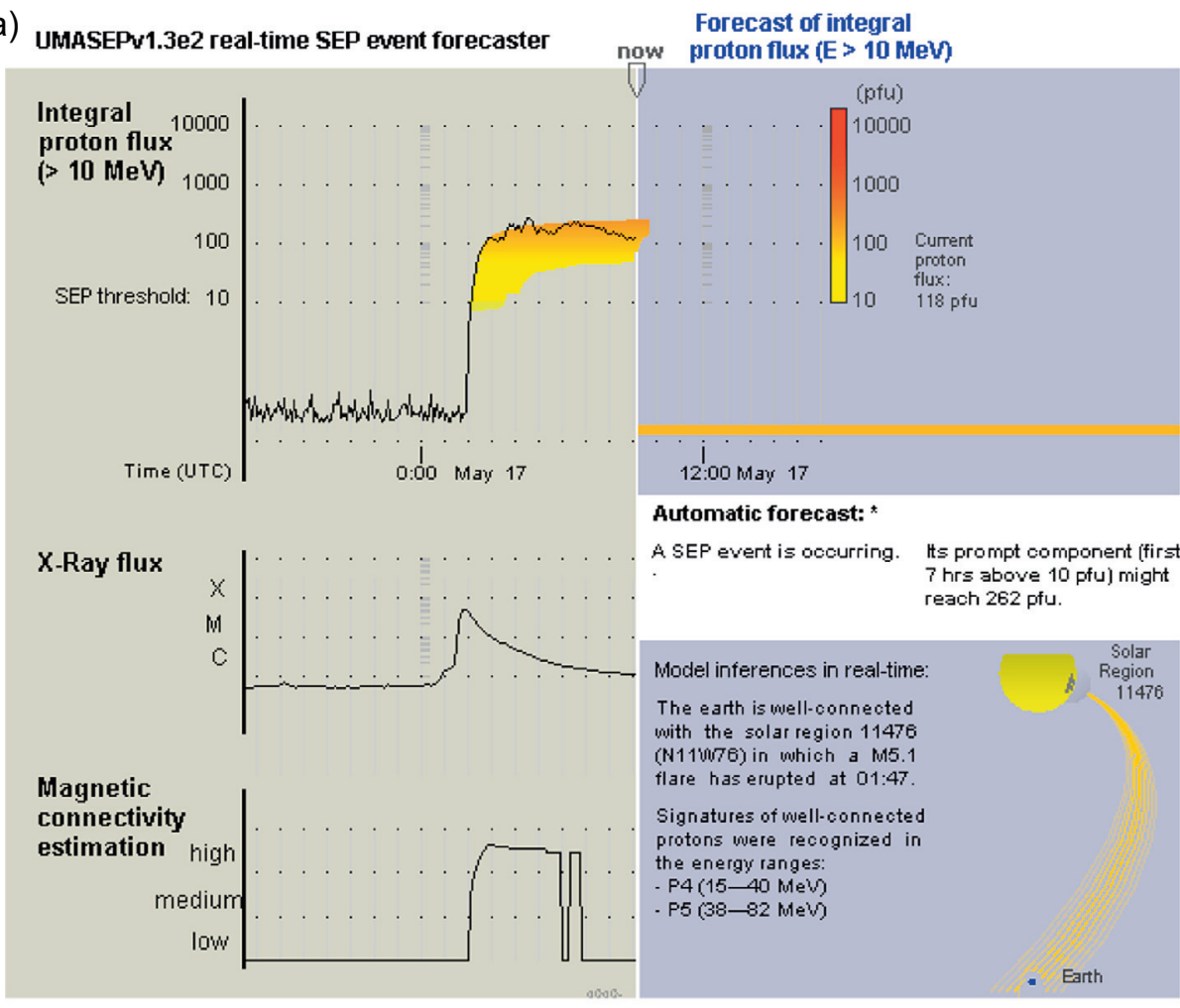

(b)
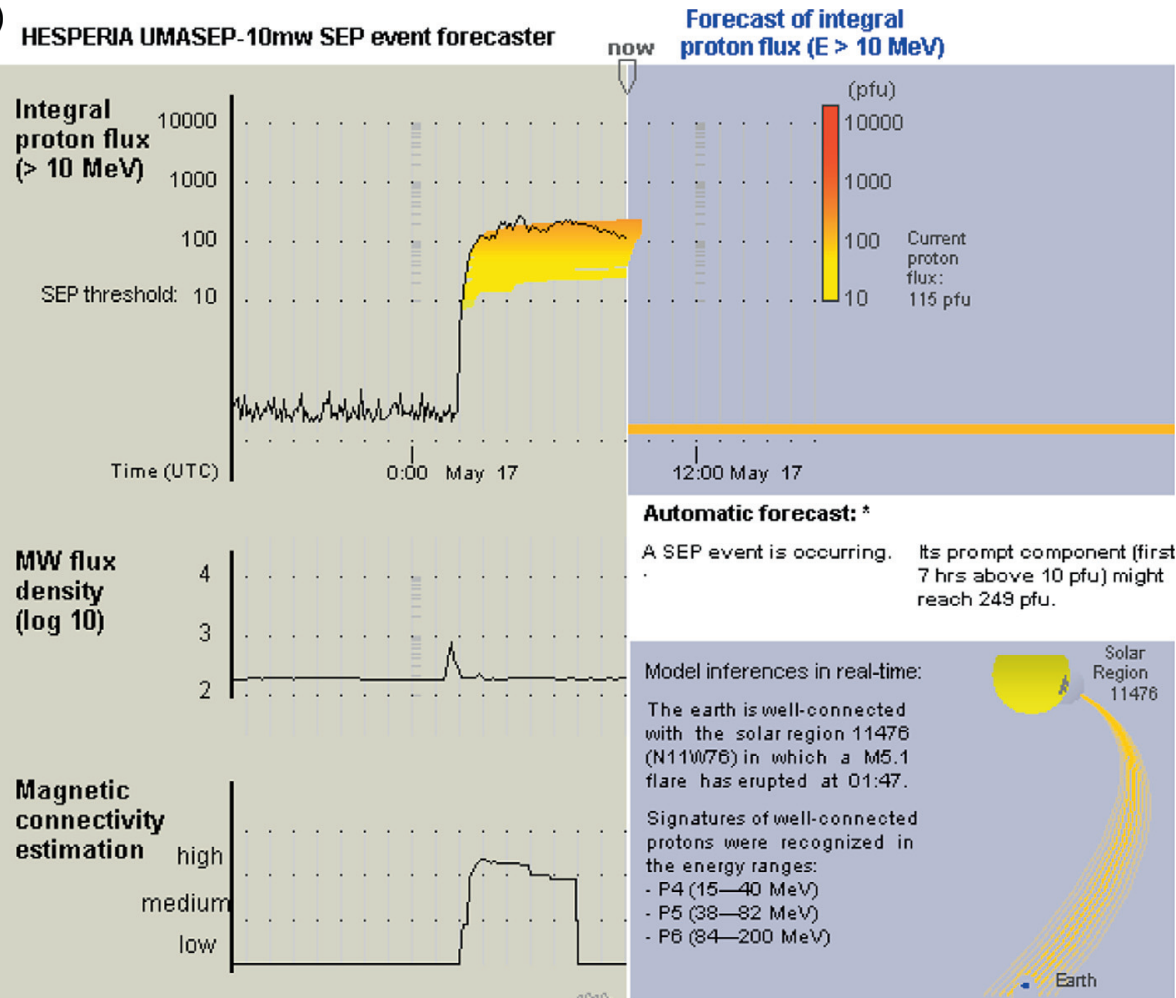

Fig. 9. UMASEP prediction web page for 2012 May 17: (a) and (b) show the SEP prediction using soft X-rays and microwaves, respectively.

to the faster rise of the microwave profile, which generated a correlation with the time derivative of the proton intensity above the similarity threshold $s_{\max }$, leading to a correct forecast of an SEP event.
We finally discuss the large SEP event of 2012 May 17, which was successfully predicted by both UMASEP-10 and UMASEP-10mw, but with a very short warning time of only $5 \mathrm{~min}$. It was missed by the original calibration of the 
UMASEP-10mw procedure: the microwave burst triggered a forecast, but this came after the SEP intensity exceeded the NOAA threshold (Fig. 9b). The short warning time is the result of a very fast arrival of the first SEPs, together with a steep rise of the time profile.

\section{Summary and discussion}

An experimental run of the UMASEP prediction scheme of the occurrence of SEP events was presented, using microwave data as an identification of connection to a solar particle source. The key findings for a 13-month period from December 2011 to December 2012 are the following:

- The probability of detection is the same as in the traditional UMASEP scheme, where the derivative of the soft $\mathrm{X}$-ray correlated with that of the SEP intensity.

- The false-alarm ratio is reduced to zero by the microwave data at both frequencies considered ( 5 and $9 \mathrm{GHz}$ ).

- The warning time obtained with the microwave light curves is slightly improved with respect to soft X-rays (30.7 vs. $26.4 \mathrm{~min})$.

The forecasting scheme using microwaves fails when the microwave emission is thermal and slowly rising (2012 June 17). Both soft X-ray based and microwave-based forecasts fail when the proton time profile rises slowly (2012 July 07). Both give only short warning times when the SEPs arrive very rapidly after the solar event (2012 May 17). Somewhat surprisingly, the forecasting seems to work on occasion even when the microwave emission is thermal bremsstrahlung, provided its rise is not too slow (2012 September 27-28). This depends of course on the calibration of the internal parameters of the UMASEP scheme, which in turn depend on the fluctuations of the detected microwave signal. Microwave bursts, be they non-thermal gyrosynchrotron emission or thermal bremsstrahlung, are rarer than thermal soft X-ray bursts. If the latter are used in SEP forecasting, an empirical threshold must be imposed on the peak flux of the soft X-ray bursts to discard the ubiquitous small events. This turns out to not be necessary for microwave bursts.

The comparatively rare occurrence of the microwave bursts probably explains the low false-alarm ratio. Spurious fluctuations of the microwave data then appear as the main problem of the method: baseline drifts due to erroneous antenna pointing or receiver instabilities, sudden jumps and slow fluctuations of the background with an amplitude well above the noise level led us to carefully calibrate the threshold associated with the minimum value of the backgroundsubtracted microwave flux density to be considered. Part of these data problems could be corrected by a more careful cleaning. But a sophisticated and reliable data analysis is hardly possible in real time. Therefore a better controlled operation of the radio instruments appears mandatory if one wants to use them for an automated prediction scheme of SEP events in an operational service.

Conclusions drawn here for the microwave emission probably pertain to hard X-rays, too. Hard X-ray time profiles are known to be similar to the time profiles of gyrosynchrotron microwaves. They do not show the thermal bremsstrahlung counterpart sometimes observed in the microwave time profiles. Since it is currently not possible to construct long uninterrupted time profiles of solar hard X-ray emission, we cannot test their predictive performance. A possible inconvenience is the sensitivity of the detectors to energetic particles, especially electrons, which contaminate observations taken outside the Earth's magnetosphere. This can be seen, for instance, in X-ray observations from the International Sun-Earth Explorer mission (ISEE-3) located at the L1 Lagrange point in Figure 1 of Kane et al. (1985). Figure 4 of Kuznetsov et al. (2011) illustrates a similar contamination effect on a gamma-ray detector in polar orbit by solar and magnetospheric protons during the 2003 October 28 event.

The radio observations exploited in the present work are carried out with rather simple patrol instruments, which monitor the whole-Sun flux density using parabolic antennas with a typical size of $1 \mathrm{~m}$. Such data are presently not provided in real time, but there is no technical obstacle to do so. If a reliable calibration and stable and reliable antenna operations can be achieved, microwave patrol observations will be a significant addition to our ability to predict the occurrence of SEP events. As attractive as microwave observations may be, they are limited to activity on the Earthward part of the solar disk or possibly just behind the western limb. The practical consequences of this limitation on the SEP impact are somewhat uncertain, because the intensity of SEPs at the Earth decreases significantly with increasing distance of the parent active region from $\mathrm{W} 100^{\circ}$. In any case the limitation is shared with present soft X-ray observations, but can be overcome in principle by placing a spacecraft in an adequate vantage point. While space-borne microwave observations are conceivable, the tool will then of course cease to be a cheap alternative to the X-rays.

Acknowledgements. This research received funding from the European Union's Horizon 2020 research and innovation programme under Grant Agreement No. 637324 (HESPERIA project). It was also supported by the Agence Nationale pour la Recherche (ANR/ASTRID, DGA) project Outils radioastronomiques pour la météorologie de l'espace (ORME, Contract No. ANR-14-ASTR-0027) and by the French space agency CNES The work is based on radio data from the RSTN network (provided through NGDC) and the Nobeyama Radio Polarimeters (NoRP). NoRP are operated by Nobeyama Radio Observatory, a branch of National Astronomical Observatory of Japan. Supporting information was provided by the Radio Monitoring web site maintained at Paris Observatory with support by CNES. The authors acknowledge detailed and helpful comments by the referees. PZ and KLK acknowledge helpful discussions with G. Trottet. The editor thanks Arik Posner and an anonymous referee for their assistance in evaluating this paper.

\section{References}

Aran, A., B. Sanahuja, and D. Lario. SOLPEN CO: a solar particle engineering code. Adv. Space Res., 37, 1240-1246, 2006, DOI: 10.1016/j.asr.2005.09.019.

Aran, A., B. Sanahuja, and D. Lario. Comparing proton fluxes of central meridian SEP events with those predicted by SOLPENCO. Adv. Space Res., 42, 1492-1499, 2008, DOI: 10.1016/j.asr.2007.08.003.

Balch, C.C. Updated verification of the space weather prediction center's solar energetic particle prediction model. Space Weather, 6, S01001, 2008, DOI: 10.1029/2007SW000337.

Belov, A. Properties of solar X-ray flares and proton event forecasting. Adv. Space Res., 43 (4), 467-473, 2009, DOI: 10.1016/j.asr.2008.08.011.

Davis, J., and M. Goadrich, The relationship between precisionrecall and ROC curves. In: W., Cohen, and A. Moore, Editors, Proceedings of the Twenty-Third International Conference on 
Machine Learning (ICML 06), vol. 148 of ACM International Conference Proceeding Series, Association for Computing Machinery, New York, NY, USA, 233-240, 2006, DOI: $10.1145 / 1143844.1143874$.

Dennis, B.R., and D.M. Zarro. The Neupert effect - what can it tell us about the impulsive and gradual phases of solar flares? Sol. Phys., 146, 177-190, 1993, DOI: 10.1007/BF00662178.

Dierckxsens, M., K. Tziotziou, S. Dalla, I. Patsou, M.S. Marsh, N.B. Crosby, O. Malandraki, and G. Tsiropoula. Relationship between solar energetic particles and properties of flares and CMEs: statistical analysis of solar cycle 23 events. Sol. Phys., 290, 841-874, 2015, DOI: 10.1007/s11207-014-0641-4.

Dresing, N., R. Gómez-Herrero, B. Heber, A. Klassen, O. Malandraki, W. Dröge, and Y. Kartavykh. Statistical survey of widely spread out solar electron events observed with STEREO and ACE with special attention to anisotropies. $A \& A$, 567, A27, 2014, DOI: 10.1051/0004-6361/201423789.

Garcia, H.A. Forecasting methods for occurrence and magnitude of proton storms with solar soft X-rays. Space Weather, 2, S02002, 2004, DOI: 10.1029/2003SW000001.

García-Rigo, A., M. Núñez, R. Qahwaji, O. Ashamari, P. Jiggens, G. Pérez, M. Hernández-Pajares, and A. Hilgers. Prediction and warning system of SEP events and solar flares for risk estimation in space launch operations. J. Space Weather Space Clim., 6 (27), A28, 2016, DOI: 10.1051/swsc/2016021.

Holman, G.D., M.J. Aschwanden, H. Aurass, M. Battaglia, P.C. Grigis, E.P. Kontar, W. Liu, P. Saint-Hilaire, and V.V. Zharkova. Implications of X-ray observations for electron acceleration and propagation in solar flares. Space Sci. Rev., 159, 107-166, 2011, DOI: 10.1007/s11214-010-9680-9.

Kahler, S.W., E.W. Cliver, and A.G. Ling. Validating the proton prediction system (PPS). J. Atmos. Solar-Terr. Phys., 69, 43-49, 2007, DOI: $10.1016 /$ j.jastp.2006.06.009.

Kane, S.R., P. Evenson, and P. Meyer. Acceleration of interplanetary solar electrons in the 1982 August 14 flare. Astrophys. J., 299, L107-L110, 1985, DOI: 10.1086/184590.

Klein, K.-L., S. Krucker, G. Lointier, and A. Kerdraon. Open magnetic flux tubes in the corona and the transport of solar energetic particles. $A \& A, \mathbf{4 8 6}, 589-596,2008$,

DOI: 10.1051/0004-6361:20079228.

Kuznetsov, S.N., V.G. Kurt, B.Y. Yushkov, K. Kudela, and V.I. Galkin. Gamma-ray and high-energy-neutron measurements on CORONAS-F during the solar flare of 28 October 2003. Sol. Phys., 268, 175-193, 2011, DOI: 10.1007/s11207-010-9669-2.

Laitinen, T., and S. Dalla. Energetic particle transport across the mean magnetic field: before diffusion. Astrophys. J., 834, 127, 2017, DOI: 10.3847/1538-4357/834/2/127.

Laurenza, M., E.W. CliverJ. Hewitt, M. Storini, A.G. Ling, C.C. Balch, and M.L. Kaiser. A technique for short-term warning of solar energetic particle events based on flare location, flare size, and evidence of particle escape. Space Weather, 7, S04008, 2009, DOI: $10.1029 / 2007$ SW000379.

Lee, M.A., R.A. Mewaldt, and J. Giacalone. Shock acceleration of ions in the heliosphere. Space Sci. Rev., 173, 247-281, 2012, DOI: $10.1007 / \mathrm{s} 11214-012-9932-y$.
Mann, G., F. Jansen, R.J. MacDowall, M.L. Kaiser, and R.G. Stone. A heliospheric density model and type III radio bursts. $A \& A$, 348, 614-620, 1999.

Marsh, M.S., S. Dalla, M. Dierckxsens, T. Laitinen, and N.B. Crosby. SPARX: a modeling system for solar energetic particle radiation space weather forecasting. Space Weather, 13, 386-394, 2015, DOI: 10.1002/2014SW001120.

Masson, S., P. Démoulin, S. Dasso, and K.-L. Klein. The interplanetary magnetic structure that guides solar relativistic particles. $A \& A, \mathbf{5 3 8}, \mathrm{A} 32,2012$, DOI: $10.1051 / 0004-6361 / 201118145$.

Nakajima, H., H. Sekiguchi, M. Sawa, K. Kai, and S. Kawashima. The radiometer and polarimeters at 80,35 , and $17 \mathrm{GHz}$ for solar observations at Nobeyama. Publ. Astron. Soc. Jpn., 37, 163-170, 1985.

Neupert, W.M. Comparison of solar X-ray line emission with microwave emission during flares. Astrophys. J., 153, L59-L64, 1968, DOI: $10.1086 / 180220$.

Núñez, M. Predicting solar energetic proton events $(E>10 \mathrm{MeV})$. Space Weather, 9, 07003, 2011, DOI: $10.1029 / 2010$ SW000640.

Núñez, M. Real-time prediction of the occurrence and intensity of the first hours of $>100 \mathrm{MeV}$ solar energetic proton events. Space Weather, 13, 807-819, 2015, DOI: $10.1002 / 2015$ SW001256.

Posner, A. Up to 1-hour forecasting of radiation hazards from solar energetic ion events with relativistic electrons. Space Weather, $\mathbf{5}$, S05001, 2007, DOI: 10.1029/2006SW000268.

Richardson, I.G., and H.V. Cane. Particle flows observed in ejecta during solar event onsets and their implication for the magnetic field topology. J. Geophys. Res., 101, 27521-27532, 1996, DOI: 10.1029/96JA02643.

Richardson, I.G., T.T. von Rosenvinge, H.V. Cane, E.R. Christian, C.M.S. Cohen, A.W. Labrador, R.A. Leske, R.A. Mewaldt, M.E. Wiedenbeck, and E.C. Stone. $>25 \mathrm{MeV}$ proton events observed by the High Energy Telescopes on the STEREO A and B spacecraft and/or at Earth during the first seven years of the STEREO mission. Sol. Phys., 289, 3059-3107, 2014, DOI: $10.1007 / \mathrm{s} 11207-014-0524-8$.

Smart, D.F., and M.A. Shea. Modeling the time-intensity profile of solar flare generated particle fluxes in the inner heliosphere. $A d v$. Space Res., 12, 303-312, 1992, DOI: 10.1016/0273-1177(92)90120-M.

Souvatzoglou, G., A. Papaioannou, H. Mavromichalaki, J. Dimitroulakos, and C. Sarlanis. Optimizing the real-time ground level enhancement alert system based on neutron monitor measurements: introducing GLE alert plus. Space Weather, 12, 633-649, 2014, DOI: 10.1002/2014SW001102.

Torii, C., Y. Tsukiji, S. Kobayashi, N. Yoshimi, H. Tanaka, and S. Enome. Full-automatic radiopolarimeters for solar patrol at microwave frequencies. Proc. Res. Inst. Atmos., Nagoya Univ., 26, 129-132, 1979.

Cite this article as: Zucca P, Núñez M \& Klein K-L. Exploring the potential of microwave diagnostics in SEP forecasting: The occurrence of SEP events. J. Space Weather Space Clim., 7, A13, 2017, DOI: 10.1051/swsc/2017011. 